Bull. Korean Math. Soc. 52 (2015), No. 2, pp. 627-647

http://dx.doi.org/10.4134/BKMS.2015.52.2.627

\title{
COVARIANT MAPS FOR THE SCHRÖDINGER-WEIL REPRESENTATION
}

\author{
JAE-HYUN YANG
}

\begin{abstract}
In this paper, we construct the Schrödinger-Weil representation of the Jacobi group associated with a positive definite symmetric real matrix of degree $m$ and find covariant maps for the Schrödinger-Weil representation.
\end{abstract}

\section{Introduction}

For a given fixed positive integer $n$, we let

$$
\mathbb{H}_{n}=\left\{\Omega \in \mathbb{C}^{(n, n)} \mid \Omega={ }^{t} \Omega, \operatorname{Im} \Omega>0\right\}
$$

be the Siegel upper half plane of degree $n$ and let

$$
S p(n, \mathbb{R})=\left\{g \in \mathbb{R}^{(2 n, 2 n)} \mid{ }^{t} g J_{n} g=J_{n}\right\}
$$

be the symplectic group of degree $n$, where $F^{(k, l)}$ denotes the set of all $k \times l$ matrices with entries in a commutative ring $F$ for two positive integers $k$ and $l,{ }^{t} M$ denotes the transpose of a matrix $M, \operatorname{Im} \Omega$ denotes the imaginary part of $\Omega$ and

$$
J_{n}=\left(\begin{array}{cc}
0 & I_{n} \\
-I_{n} & 0
\end{array}\right) .
$$

Here $I_{n}$ denotes the identity matrix of degree $n$. We see that $S p(n, \mathbb{R})$ acts on $\mathbb{H}_{n}$ transitively by

$$
g \cdot \Omega=(A \Omega+B)(C \Omega+D)^{-1},
$$

where $g=\left(\begin{array}{ll}A & B \\ C & D\end{array}\right) \in S p(n, \mathbb{R})$ and $\Omega \in \mathbb{H}_{n}$.

For two positive integers $n$ and $m$, we consider the Heisenberg group

$$
H_{\mathbb{R}}^{(n, m)}=\left\{(\lambda, \mu ; \kappa) \mid \lambda, \mu \in \mathbb{R}^{(m, n)}, \kappa \in \mathbb{R}^{(m, m)}, \kappa+\mu^{t} \lambda \text { symmetric }\right\}
$$

Received April 25, 2014; Revised August 19, 2014

2010 Mathematics Subject Classification. Primary 11F27, 11F50.

Key words and phrases. the Schrödinger representation, the Schrödinger-Weil representation, covariant maps, Jacobi forms.

The author was supported by Basic Science Program through the National Research Foundation of Korea (NRF) funded by the Ministry of Education, Science and Technology (47724-1). 
endowed with the following multiplication law

$$
(\lambda, \mu ; \kappa) \circ\left(\lambda^{\prime}, \mu^{\prime} ; \kappa^{\prime}\right)=\left(\lambda+\lambda^{\prime}, \mu+\mu^{\prime} ; \kappa+\kappa^{\prime}+\lambda^{t} \mu^{\prime}-\mu^{t} \lambda^{\prime}\right) .
$$

We let

$$
G^{J}=S p(n, \mathbb{R}) \ltimes H_{\mathbb{R}}^{(n, m)} \quad \text { (semi-direct product) }
$$

be the Jacobi group endowed with the following multiplication law

$$
(g,(\lambda, \mu ; \kappa)) \cdot\left(g^{\prime},\left(\lambda^{\prime}, \mu^{\prime} ; \kappa^{\prime}\right)\right)=\left(g g^{\prime},\left(\widetilde{\lambda}+\lambda^{\prime}, \widetilde{\mu}+\mu^{\prime} ; \kappa+\kappa^{\prime}+\widetilde{\lambda}^{t} \mu^{\prime}-\widetilde{\mu}^{t} \lambda^{\prime}\right)\right)
$$

with $g, g^{\prime} \in S p(n, \mathbb{R}),(\lambda, \mu ; \kappa),\left(\lambda^{\prime}, \mu^{\prime} ; \kappa^{\prime}\right) \in H_{\mathbb{R}}^{(n, m)}$ and $(\widetilde{\lambda}, \widetilde{\mu})=(\lambda, \mu) g^{\prime}$. We let $\Gamma_{n}=S p(n, \mathbb{Z})$ be the Siegel modular group of degree $n$. We let

$$
\Gamma_{n}^{J}=\Gamma_{n} \ltimes H_{\mathbb{Z}}^{(n, m)}
$$

be the Jacobi modular group, where

$$
H_{\mathbb{Z}}^{(n, m)}:=\left\{(\lambda, \mu ; \kappa) \in H_{\mathbb{R}}^{(n, m)} \mid \lambda, \mu, \kappa \text { are integral }\right\}
$$

is the discrete subgroup of $H_{\mathbb{R}}^{(n, m)}$. Then we have the natural action of $G^{J}$ on the Siegel-Jacobi space $\mathbb{H}_{n, m}:=\mathbb{H}_{n} \times \mathbb{C}^{(m, n)}$ defined by

$$
(g,(\lambda, \mu ; \kappa)) \cdot(\Omega, Z)=\left((A \Omega+B)(C \Omega+D)^{-1},(Z+\lambda \Omega+\mu)(C \Omega+D)^{-1}\right),
$$

where $g=\left(\begin{array}{cc}A & B \\ C & D\end{array}\right) \in S p(n, \mathbb{R}),(\lambda, \mu ; \kappa) \in H_{\mathbb{R}}^{(n, m)}$ and $(\Omega, Z) \in \mathbb{H}_{n, m}$. Thus $\mathbb{H}_{n, m}$ is a homogeneous Kähler space which is not symmetric. In fact, $\mathbb{H}_{n, m}$ is biholomorphic to the homogeneous space $G^{J} / K^{J}$, where $K^{J} \cong U(n) \times S(m, \mathbb{R})$. Here $U(n)$ denotes the unitary group of degree $n$ and $S(m, \mathbb{R})$ denote the abelian additive group consisting of all $m \times m$ symmetric real matrices. We refer to $[1,2,7,20,21,23,24,28,29,30,31,33,34,35]$ for more details on materials related to the Siegel-Jacobi space, e.g., Jacobi forms, invariant metrics, invariant differential operators and Maass-Jacobi forms.

The Weil representation for a symplectic group was first introduced by A. Weil in [18] to reformulate Siegel's analytic theory of quadratic forms (cf. [14]) in terms of the group theoretical language. It is well known that the Weil representation plays a central role in the study of the transformation behaviors of theta series. Whenever we study the transformation formulas of theta series or Siegel modular forms of half integral weights, we are troubled by the ambiguity of the factor $\operatorname{det}(C \Omega+D)^{1 / 2}$ in its signature. This means that we should consider the transformation formula on a non-trivial two-fold covering group of a symplectic group. In his paper [16], Takase removed the ambiguity of the factor $\operatorname{det}(C \Omega+D)^{1 / 2}$ by constructing the right explicit automorphy factor $J_{1 / 2}$ of weight $1 / 2$ for $S p(n, \mathbb{R})_{*}$ on $\mathbb{H}_{n}$ :

$$
J_{1 / 2}: S p(n, \mathbb{R})_{*} \times \mathbb{H}_{n} \longrightarrow \mathbb{C}^{\times} .
$$


Here $S p(n, \mathbb{R})_{*}$ is the two-fold covering group of $S p(n, \mathbb{R})$ in the sense of a real Lie group. See (4.15) for the precise definition. $J_{1 / 2}$ is real analytic on $S p(n, \mathbb{R})_{*}$, holomorphic on $\mathbb{H}_{n}$ and satisfies the relation

$$
J_{1 / 2}\left(g_{*}, \Omega\right)^{2}=\operatorname{det}(C \Omega+D),
$$

where $g_{*}=(g, \epsilon) \in S p(n, \mathbb{R})_{*}$ with $g=\left(\begin{array}{cc}A & B \\ C & D\end{array}\right) \in S p(n, \mathbb{R})$. Using the automorphy factor $J_{1 / 2}$ of weight $1 / 2$, Takase expressed the transformation formula of theta series without ambiguity of $\operatorname{det}(C \Omega+D)^{1 / 2}$. Moreover he decomposed the automorphy factor

$$
j(\gamma, \Omega)=\frac{\vartheta(\gamma \cdot \Omega)}{\vartheta(\Omega)}
$$

with a standard theta series $\vartheta(\Omega)$ into a product of a character and the automorphy factor $J_{1 / 2}\left(\gamma_{*}, \Omega\right)$.

In this paper, we construct the Schrödinger-Weil representation $\omega_{\mathcal{M}}$ of the Jacobi group $G^{J}$ associated with a positive definite symmetric real matrix $\mathcal{M}$ of degree $n$. We construct the automorphy factor $J_{\mathcal{M}}^{*}$ (see Formula (5.3)) of the two-fold covering group of $G^{J}$ using the automorphy factor $J_{1 / 2}$ of Takase. And we find the covariant maps (cf. [9], pp. 123-125) for the Schrödinger-Weil representation $\omega_{\mathcal{M}}$ and the automorphy factor $J_{\mathcal{M}}^{*}$ satisfying the covariant relation (5.4).

This paper is organized as follows. In Section 2, we review the Schrödinger representation of the Heisenberg group $H_{\mathbb{R}}^{(n, m)}$ associated with a nonzero symmetric real matrix of degree $m$ which is formulated in $[19,22,27]$. In Section 3 , we define the Schrödinger-Weil representation $\omega_{\mathcal{M}}$ of the Jacobi group $G^{J}$ associated with a symmetric positive definite matrix $\mathcal{M}$ and provide some of the actions of $\omega_{\mathcal{M}}$ on the representation space $L^{2}\left(\mathbb{R}^{(m, n)}\right)$ explicitly. In Section 4, we review Jacobi forms of integral weight, Siegel modular forms of half integral weight, and the automorphy factor $J_{1 / 2}$ of weight $1 / 2$ for the metaplectic group $S p(n, \mathbb{R})_{*}$ on $\mathbb{H}_{n}$ constructed by Takase (cf. [16]). In Section 5, we construct the automorphy factor $J_{\mathcal{M}}^{*}$ of the two-fold covering group of $G^{J}$ using the automorphy factor $J_{1 / 2}$ of Takase and then find covariant maps for the Schrödinger-Weil representation $\omega_{\mathcal{M}}$. As an application, we construct a Jacobi form of half integral weight and index $\mathcal{M}$.

Notations: We denote by $\mathbb{Z}, \mathbb{R}$ and $\mathbb{C}$ the ring of integers, the field of real numbers and the field of complex numbers respectively. $\mathbb{C}^{\times}$denotes the multiplicative group of nonzero complex numbers and $\mathbb{Z}^{\times}$denotes the set of all nonzero integers. $T$ denotes the multiplicative group of complex numbers of modulus one. The symbol ":=" means that the expression on the right is the definition of that on the left. For two positive integers $k$ and $l, F^{(k, l)}$ denotes the set of all $k \times l$ matrices with entries in a commutative ring $F$. For a square matrix $A \in F^{(k, k)}$ of degree $k, \sigma(A)$ denotes the trace of $A$. For any $M \in F^{(k, l)},{ }^{t} M$ denotes the transpose of a matrix $M . I_{n}$ denotes the identity matrix of degree $n$. We put $i=\sqrt{-1}$. For $z \in \mathbb{C}$, we define $z^{1 / 2}=\sqrt{z}$ so that 
$-\pi / 2<\arg \left(z^{1 / 2}\right) \leqq \pi / 2$. Further we put $z^{\kappa / 2}=\left(z^{1 / 2}\right)^{\kappa}$ for every $\kappa \in \mathbb{Z}$. For a positive integer $m$ we denote by $S(m, F)$ the additive group consisting of all $m \times m$ symmetric matrices with coefficients in a commutative ring $F$.

\section{The Schrödinger representation}

First of all, we observe that $H_{\mathbb{R}}^{(n, m)}$ is a 2-step nilpotent Lie group. The inverse of an element $(\lambda, \mu ; \kappa) \in H_{\mathbb{R}}^{(n, m)}$ is given by

$$
(\lambda, \mu ; \kappa)^{-1}=\left(-\lambda,-\mu ;-\kappa+\lambda^{t} \mu-\mu^{t} \lambda\right) .
$$

Now we set

$$
[\lambda, \mu ; \kappa]=(0, \mu ; \kappa) \circ(\lambda, 0 ; 0)=\left(\lambda, \mu ; \kappa-\mu^{t} \lambda\right) .
$$

Then $H_{\mathbb{R}}^{(n, m)}$ may be regarded as a group equipped with the following multiplication

$$
[\lambda, \mu ; \kappa] \diamond\left[\lambda_{0}, \mu_{0} ; \kappa_{0}\right]=\left[\lambda+\lambda_{0}, \mu+\mu_{0} ; \kappa+\kappa_{0}+\lambda^{t} \mu_{0}+\mu_{0}{ }^{t} \lambda\right] .
$$

The inverse of $[\lambda, \mu ; \kappa] \in H_{\mathbb{R}}^{(n, m)}$ is given by

$$
[\lambda, \mu ; \kappa]^{-1}=\left[-\lambda,-\mu ;-\kappa+\lambda^{t} \mu+\mu^{t} \lambda\right] .
$$

We set

$$
L=\left\{[0, \mu ; \kappa] \in H_{\mathbb{R}}^{(n, m)} \mid \mu \in \mathbb{R}^{(m, n)}, \kappa={ }^{t} \kappa \in \mathbb{R}^{(m, m)}\right\} .
$$

Then $L$ is a commutative normal subgroup of $H_{\mathbb{R}}^{(n, m)}$. Let $\widehat{L}$ be the Pontrajagin dual of $L$, i.e., the commutative group consisting of all unitary characters of $L$. Then $\widehat{L}$ is isomorphic to the additive group $\mathbb{R}^{(m, n)} \times S(m, \mathbb{R})$ via the canonical pairing

$$
\langle a, \hat{a}\rangle=e^{2 \pi i \sigma\left(\hat{\mu}^{t} \mu+\hat{\kappa} \kappa\right)}, \quad a=[0, \mu ; \kappa] \in L, \hat{a}=(\hat{\mu}, \hat{\kappa}) \in \widehat{L},
$$

where $S(m, \mathbb{R})$ denotes the space of all symmetric $m \times m$ real matrices.

We put

$$
S=\left\{[\lambda, 0 ; 0] \in H_{\mathbb{R}}^{(n, m)} \mid \lambda \in \mathbb{R}^{(m, n)}\right\} \cong \mathbb{R}^{(m, n)} .
$$

Then $S$ acts on $L$ as follows:

$$
[\lambda, 0 ; 0] *[0, \mu ; \kappa]:=\left[0, \mu ; \kappa+\lambda^{t} \mu+\mu^{t} \lambda\right], \quad[\lambda, 0,0] \in S,[0, \mu ; \kappa] \in L .
$$

We see that the Heisenberg group $\left(H_{\mathbb{R}}^{(n, m)}, \diamond\right)$ is isomorphic to the semi-direct product $S \ltimes L$ of $S$ and $L$ whose multiplication law is defined by

$$
\begin{aligned}
& ([\lambda, 0 ; 0],[0, \mu ; \kappa]) \star\left(\left[\lambda_{0}, 0 ; 0\right],\left[0, \mu_{0} ; \kappa_{0}\right]\right) \\
:= & \left(\left[\lambda+\lambda_{0}, 0 ; 0\right],\left[0, \mu+\mu_{0} ; \kappa+\kappa_{0}+\lambda^{t} \mu_{0}+\mu_{0}{ }^{t} \lambda\right]\right) .
\end{aligned}
$$

On the other hand, $S$ acts on $\widehat{L}$ by

$$
[\lambda, 0 ; 0] \bullet(\hat{\mu}, \hat{\kappa})=(\hat{\mu}+2 \hat{\kappa} \lambda, \hat{\kappa}),
$$


where $[\lambda, 0 ; 0] \in S,(\hat{\mu}, \hat{\kappa}) \in \widehat{L}$ with $\hat{\mu} \in \mathbb{R}^{(m, n)}$ and $\hat{\kappa} \in S(m, \mathbb{R})$. Then we have the following relation

$$
\langle[\lambda, 0 ; 0] *[0, \mu ; \kappa],(\hat{\mu}, \hat{\kappa})\rangle=\langle[0, \mu ; \kappa],[\lambda, 0 ; 0] \bullet(\hat{\mu}, \hat{\kappa})\rangle,
$$

where $[\lambda, 0 ; 0] \in S,[0, \mu ; \kappa] \in L$ and $(\hat{\mu}, \hat{\kappa}) \in \widehat{L}$.

We have three types of $S$-orbits in $\widehat{L}$.

TyPe I. Let $\hat{\kappa} \in S(m, \mathbb{R})$ be nondegenerate. The $S$-orbit of $(0, \hat{\kappa}) \in \widehat{L}$ is given by

$$
\widehat{\mathcal{O}}_{\hat{\kappa}}=\left\{(2 \hat{\kappa} \lambda, \hat{\kappa}) \in \widehat{L} \mid \lambda \in \mathbb{R}^{(m, n)}\right\} \cong \mathbb{R}^{(m, n)} .
$$

TYPE II. Let $(\hat{\mu}, \hat{\kappa}) \in \mathbb{R}^{(m, n)} \times S(m, \mathbb{R})$ with $\hat{\mu} \in \mathbb{R}^{(m, n)}, \hat{\kappa} \in S(m, \mathbb{R})$ and degenerate $\hat{\kappa} \neq 0$. Then

$$
\widehat{\mathcal{O}}_{(\hat{\mu}, \hat{\kappa})}=\left\{(\hat{\mu}+2 \hat{\kappa} \lambda, \hat{\kappa}) \mid \lambda \in \mathbb{R}^{(m, n)}\right\} \varsubsetneqq \mathbb{R}^{(m, n)} \times\{\hat{\kappa}\} .
$$

Type III. Let $\hat{y} \in \mathbb{R}^{(m, n)}$. The $S$-orbit $\widehat{\mathcal{O}}_{\hat{y}}$ of $(\hat{y}, 0)$ is given by

$$
\widehat{\mathcal{O}}_{\hat{y}}=\{(\hat{y}, 0)\} .
$$

We have the following disjoint union

$$
\widehat{L}=\left(\bigcup_{\substack{\hat{\kappa} \in S(m, \mathbb{R}) \\ \hat{\kappa} \\ \text { nondegenerate }}} \widehat{\mathcal{O}}_{\hat{\kappa}}\right) \bigcup\left(\bigcup_{\hat{y} \in \mathbb{R}^{(m, n)}} \widehat{\mathcal{O}}_{\hat{y}}\right) \bigcup\left(\bigcup_{\substack{\hat{\mu}, \hat{\kappa}) \in \mathbb{R}^{(m, n)} \times S(m, \mathbb{R}) \\ \hat{\kappa} \neq 0 \text { degenerate }}} \widehat{\mathcal{O}}_{(\hat{\mu}, \hat{\kappa})}\right)
$$

as a set. The stabilizer $S_{\hat{\kappa}}$ of $S$ at $(0, \hat{\kappa})$ with nondegenerate $\hat{\kappa}$ is given by

$$
S_{\hat{\kappa}}=\{0\} .
$$

And the stabilizer $S_{\hat{y}}$ of $S$ at $(\hat{y}, 0)$ is given by

$$
S_{\hat{y}}=\left\{[\lambda, 0 ; 0] \mid \lambda \in \mathbb{R}^{(m, n)}\right\}=S \cong \mathbb{R}^{(m, n)} .
$$

In this section, for the present being we set $H=H_{\mathbb{R}}^{(n, m)}$ for brevity. We see that $L$ is a closed, commutative normal subgroup of $H$. Since $(\lambda, \mu ; \kappa)=$ $\left(0, \mu ; \kappa+\mu^{t} \lambda\right) \circ(\lambda, 0 ; 0)$ for $(\lambda, \mu ; \kappa) \in H$, the homogeneous space $X=L \backslash H$ can be identified with $\mathbb{R}^{(m, n)}$ via

$$
L h=L \circ(\lambda, 0 ; 0) \longmapsto \lambda, \quad h=(\lambda, \mu ; \kappa) \in H .
$$

We observe that $H$ acts on $X$ by

$$
(L h) \cdot h_{0}=L\left(\lambda+\lambda_{0}, 0 ; 0\right)=\lambda+\lambda_{0},
$$

where $h=(\lambda, \mu ; \kappa) \in H$ and $h_{0}=\left(\lambda_{0}, \mu_{0} ; \kappa_{0}\right) \in H$.

If $h=(\lambda, \mu ; \kappa) \in H$, according to the Mackey decomposition of $h=l_{h} \circ s_{h}$ with $l_{h} \in L$ and $s_{h} \in S$, (cf. [10]) we have

$$
l_{h}=\left(0, \mu ; \kappa+\mu^{t} \lambda\right), \quad s_{h}=(\lambda, 0 ; 0) .
$$


Thus if $h_{0}=\left(\lambda_{0}, \mu_{0} ; \kappa_{0}\right) \in H$, then we have

$$
s_{h} \circ h_{0}=(\lambda, 0 ; 0) \circ\left(\lambda_{0}, \mu_{0} ; \kappa_{0}\right)=\left(\lambda+\lambda_{0}, \mu_{0} ; \kappa_{0}+\lambda^{t} \mu_{0}\right)
$$

and so

$$
l_{s_{h} \circ h_{0}}=\left(0, \mu_{0} ; \kappa_{0}+\mu_{0}{ }^{t} \lambda_{0}+\lambda^{t} \mu_{0}+\mu_{0}{ }^{t} \lambda\right) .
$$

For a real symmetric matrix $c={ }^{t} c \in S(m, \mathbb{R})$ with $c \neq 0$, we consider the unitary character $\chi_{c}$ of $L$ defined by

$$
\chi_{c}((0, \mu ; \kappa))=e^{\pi i \sigma(c \kappa)}, \quad(0, \mu ; \kappa) \in L .
$$

Then the representation $\mathscr{W}_{c}=\operatorname{Ind}_{L}^{H} \chi_{c}$ of $H$ induced from $\chi_{c}$ is realized on the Hilbert space $H\left(\chi_{c}\right)=L^{2}(X, d \dot{h}, \mathbb{C}) \cong L^{2}\left(\mathbb{R}^{(m, n)}, d \xi\right)$ as follows. If $h_{0}=$ $\left(\lambda_{0}, \mu_{0} ; \kappa_{0}\right) \in H$ and $x=L h \in X$ with $h=(\lambda, \mu ; \kappa) \in H$, we have

$$
\left(\mathscr{W}_{c}\left(h_{0}\right) f\right)(x)=\chi_{c}\left(l_{s_{h} \circ h_{0}}\right) f\left(x h_{0}\right), \quad f \in H\left(\chi_{c}\right) .
$$

According to (2.1) and (2.2), we can describe Formula (2.3) more explicitly as follows.

$$
\left[\mathscr{W}_{c}\left(h_{0}\right) f\right](\lambda)=e^{\pi i \sigma\left\{c\left(\kappa_{0}+\mu_{0}{ }^{t} \lambda_{0}+2 \lambda^{t} \mu_{0}\right)\right\}} f\left(\lambda+\lambda_{0}\right),
$$

where $h_{0}=\left(\lambda_{0}, \mu_{0} ; \kappa_{0}\right) \in H$ and $\lambda \in \mathbb{R}^{(m, n)}$. Here we identified $x=L h$ (resp. $\left.x h_{0}=L h h_{0}\right)$ with $\lambda$ (resp. $\lambda+\lambda_{0}$ ). The induced representation $\mathscr{W}_{c}$ is called the Schrödinger representation of $H$ associated with $\chi_{c}$. Thus $\mathscr{W}_{c}$ is a monomial representation.

Theorem 2.1. Let $c$ be a positive definite symmetric real matrix of degree $m$. Then the Schrödinger representation $\mathscr{W}_{c}$ of $H$ is irreducible.

Proof. The proof can be found in [19], Theorem 3.

Remark 2.1. We refer to [19, 22, 25, 26, 27, 32] for more representations of the Heisenberg group $H_{\mathbb{R}}^{(n, m)}$ and their related topics.

\section{The Schrödinger-Weil representation}

Throughout this section we assume that $\mathcal{M}$ is a positive definite symmetric real $m \times m$ matrix. We consider the Schrödinger representation $\mathscr{W}_{\mathcal{M}}$ of the Heisenberg group $H_{\mathbb{R}}^{(n, m)}$ with the central character $\mathscr{W}_{\mathcal{M}}((0,0 ; \kappa))=$ $\chi_{\mathcal{M}}((0,0 ; \kappa))=e^{\pi i \sigma(\mathcal{M} \kappa)}, \kappa \in S(m, \mathbb{R})(\mathrm{cf} .(2.2))$. We note that the symplectic group $S p(n, \mathbb{R})$ acts on $H_{\mathbb{R}}^{(n, m)}$ by conjugation inside $G^{J}$. For a fixed element $g \in S p(n, \mathbb{R})$, the irreducible unitary representation $\mathscr{W}_{\mathcal{M}}^{g}$ of $H_{\mathbb{R}}^{(n, m)}$ defined by

$$
\mathscr{W}_{\mathcal{M}}^{g}(h)=\mathscr{W}_{\mathcal{M}}\left(g h g^{-1}\right), \quad h \in H_{\mathbb{R}}^{(n, m)}
$$

has the property that

$$
\mathscr{W}_{\mathcal{M}}^{g}((0,0 ; \kappa))=\mathscr{W}_{\mathcal{M}}((0,0 ; \kappa))=e^{\pi i \sigma(\mathcal{M} \kappa)} \operatorname{Id}_{H\left(\chi_{\mathcal{M}}\right)}, \quad \kappa \in S(m, \mathbb{R}) .
$$


Here $\operatorname{Id}_{H\left(\chi_{\mathcal{M}}\right)}$ denotes the identity operator on the Hilbert space $H\left(\chi_{\mathcal{M}}\right)$. According to Stone-von Neumann theorem, there exists a unitary operator $R_{\mathcal{M}}(g)$ on $H\left(\chi_{\mathcal{M}}\right)$ with $R_{\mathcal{M}}\left(I_{2 n}\right)=\operatorname{Id}_{H\left(\chi_{\mathcal{M}}\right)}$ such that

$$
R_{\mathcal{M}}(g) \mathscr{W}_{\mathcal{M}}(h)=\mathscr{W}_{\mathcal{M}}^{g}(h) R_{\mathcal{M}}(g) \quad \text { for all } h \in H_{\mathbb{R}}^{(n, m)} .
$$

We observe that $R_{\mathcal{M}}(g)$ is determined uniquely up to a scalar of modulus one.

From now on, for brevity, we put $G=S p(n, \mathbb{R})$. According to Schur's lemma, we have a map $c_{\mathcal{M}}: G \times G \longrightarrow T$ satisfying the relation

$$
R_{\mathcal{M}}\left(g_{1} g_{2}\right)=c_{\mathcal{M}}\left(g_{1}, g_{2}\right) R_{\mathcal{M}}\left(g_{1}\right) R_{\mathcal{M}}\left(g_{2}\right) \quad \text { for all } g_{1}, g_{2} \in G \text {. }
$$

We recall that $T$ denotes the multiplicative group of complex numbers of modulus one. Therefore $R_{\mathcal{M}}$ is a projective representation of $G$ on $H\left(\chi_{\mathcal{M}}\right)$ and $c_{\mathcal{M}}$ defines the cocycle class in $H^{2}(G, T)$. The cocycle $c_{\mathcal{M}}$ yields the central extension $G_{\mathcal{M}}$ of $G$ by $T$. The group $G_{\mathcal{M}}$ is a set $G \times T$ equipped with the following multiplication

$$
\left(g_{1}, t_{1}\right) \cdot\left(g_{2}, t_{2}\right)=\left(g_{1} g_{2}, t_{1} t_{2} c_{\mathcal{M}}\left(g_{1}, g_{2}\right)^{-1}\right), \quad g_{1}, g_{2} \in G, t_{1}, t_{2} \in T .
$$

We see immediately that the map $\widetilde{R}_{\mathcal{M}}: G_{\mathcal{M}} \longrightarrow G L\left(H\left(\chi_{\mathcal{M}}\right)\right)$ defined by

$$
\widetilde{R}_{\mathcal{M}}(g, t)=t R_{\mathcal{M}}(g) \quad \text { for all }(g, t) \in G_{\mathcal{M}}
$$

is a true representation of $G_{\mathcal{M}}$. As in Section 1.7 in [9], we can define the map $s_{\mathcal{M}}: G \longrightarrow T$ satisfying the relation

$$
c_{\mathcal{M}}\left(g_{1}, g_{2}\right)^{2}=s_{\mathcal{M}}\left(g_{1}\right)^{-1} s_{\mathcal{M}}\left(g_{2}\right)^{-1} s_{\mathcal{M}}\left(g_{1} g_{2}\right) \quad \text { for all } g_{1}, g_{2} \in G .
$$

Thus we see that

$$
G_{2, \mathcal{M}}=\left\{(g, t) \in G_{\mathcal{M}} \mid t^{2}=s_{\mathcal{M}}(g)^{-1}\right\}
$$

is the metaplectic group associated with $\mathcal{M}$ that is a two-fold covering group of $G$. The restriction $R_{2, \mathcal{M}}$ of $\widetilde{R}_{\mathcal{M}}$ to $G_{2, \mathcal{M}}$ is the Weil representation of $G$ associated with $\mathcal{M}$.

If we identify $h=(\lambda, \mu ; \kappa) \in H_{\mathbb{R}}^{(n, m)}($ resp. $g \in S p(n, \mathbb{R}))$ with $\left(I_{2 n},(\lambda, \mu ; \kappa)\right)$ $\in G^{J}$ (resp. $\left.(g,(0,0 ; 0)) \in G^{J}\right)$, every element $\tilde{g}$ of $G^{J}$ can be written as $\tilde{g}=h g$ with $h \in H_{\mathbb{R}}^{(n, m)}$ and $g \in S p(n, \mathbb{R})$. In fact,

$$
(g,(\lambda, \mu ; \kappa))=\left(I_{2 n},\left((\lambda, \mu) g^{-1} ; \kappa\right)\right)(g,(0,0 ; 0))=\left((\lambda, \mu) g^{-1} ; \kappa\right) \cdot g .
$$

Therefore we define the projective representation $\pi_{\mathcal{M}}$ of the Jacobi group $G^{J}$ with cocycle $c_{\mathcal{M}}\left(g_{1}, g_{2}\right)$ by

$$
\pi_{\mathcal{M}}(h g)=\mathscr{W}_{\mathcal{M}}(h) R_{\mathcal{M}}(g), \quad h \in H_{\mathbb{R}}^{(n, m)}, g \in G .
$$

Indeed, since $H_{\mathbb{R}}^{(n, m)}$ is a normal subgroup of $G^{J}$, for any $h_{1}, h_{2} \in H_{\mathbb{R}}^{(n, m)}$ and $g_{1}, g_{2} \in G$,

$$
\begin{aligned}
\pi_{\mathcal{M}}\left(h_{1} g_{1} h_{2} g_{2}\right) & =\pi_{\mathcal{M}}\left(h_{1} g_{1} h_{2} g_{1}^{-1} g_{1} g_{2}\right) \\
& =\mathscr{W}_{\mathcal{M}}\left(h_{1}\left(g_{1} h_{2} g_{1}^{-1}\right)\right) R_{\mathcal{M}}\left(g_{1} g_{2}\right) \\
& =c_{\mathcal{M}}\left(g_{1}, g_{2}\right) \mathscr{W}_{\mathcal{M}}\left(h_{1}\right) \mathscr{W}_{\mathcal{M}}^{g_{1}}\left(h_{2}\right) R_{\mathcal{M}}\left(g_{1}\right) R_{\mathcal{M}}\left(g_{2}\right)
\end{aligned}
$$




$$
\begin{aligned}
& =c_{\mathcal{M}}\left(g_{1}, g_{2}\right) \mathscr{W}_{\mathcal{M}}\left(h_{1}\right) R_{\mathcal{M}}\left(g_{1}\right) \mathscr{W}_{\mathcal{M}}\left(h_{2}\right) R_{\mathcal{M}}\left(g_{2}\right) \\
& =c_{\mathcal{M}}\left(g_{1}, g_{2}\right) \pi_{\mathcal{M}}\left(h_{1} g_{1}\right) \pi_{\mathcal{M}}\left(h_{2} g_{2}\right) .
\end{aligned}
$$

We let

$$
G_{\mathcal{M}}^{J}=G_{\mathcal{M}} \ltimes H_{\mathbb{R}}^{(n, m)}
$$

be the semidirect product of $G_{\mathcal{M}}$ and $H_{\mathbb{R}}^{(n, m)}$ with the multiplication law

$$
\begin{aligned}
& \left(\left(g_{1}, t_{1}\right),\left(\lambda_{1}, \mu_{1} ; \kappa_{1}\right)\right) \cdot\left(\left(g_{2}, t_{2}\right),\left(\lambda_{2}, \mu_{2} ; \kappa_{2}\right)\right) \\
= & \left(\left(g_{1}, t_{1}\right)\left(g_{2}, t_{2}\right),\left(\tilde{\lambda}+\lambda_{2}, \tilde{\mu}+\mu_{2} ; \kappa_{1}+\kappa_{2}+\tilde{\lambda}^{t} \mu_{2}-\tilde{\mu}^{t} \lambda_{2}\right)\right),
\end{aligned}
$$

where $\left(g_{1}, t_{1}\right),\left(g_{2}, t_{2}\right) \in G_{\mathcal{M}},\left(\lambda_{1}, \mu_{1} ; \kappa_{1}\right),\left(\lambda_{2}, \mu_{2} ; \kappa_{2}\right) \in H_{\mathbb{R}}^{(n, m)}$ and $(\tilde{\lambda}, \tilde{\mu})=$ $(\lambda, \mu) g_{2}$. If we identify $h=(\lambda, \mu ; \kappa) \in H_{\mathbb{R}}^{(n, m)}\left(\operatorname{resp.}(g, t) \in G_{\mathcal{M}}\right)$ with $\left(\left(I_{2 n}, 1\right),(\lambda, \mu ; \kappa)\right) \in G_{\mathcal{M}}^{J}$ (resp. $\left.((g, t),(0,0 ; 0)) \in G_{\mathcal{M}}^{J}\right)$, we see easily that every element $((g, t),(\lambda, \mu ; \kappa))$ of $G_{\mathcal{M}}^{J}$ can be expressed as

$((g, t),(\lambda, \mu ; \kappa))=\left(\left(I_{2 n}, 1\right),\left((\lambda, \mu) g^{-1} ; \kappa\right)\right)((g, t),(0,0 ; 0))=\left((\lambda, \mu) g^{-1} ; \kappa\right)(g, t)$.

Now we can define the true representation $\widetilde{\omega}_{\mathcal{M}}$ of $G_{\mathcal{M}}^{J}$ by

$(3.8) \widetilde{\omega}_{\mathcal{M}}(h \cdot(g, t))=t \pi_{\mathcal{M}}(h g)=t \mathscr{W}_{\mathcal{M}}(h) R_{\mathcal{M}}(g), \quad h \in H_{\mathbb{R}}^{(n, m)},(g, t) \in G_{\mathcal{M}}$.

Indeed, since $H_{\mathbb{R}}^{(n, m)}$ is a normal subgroup of $G_{\mathcal{M}}^{J}$,

$$
\begin{aligned}
& \widetilde{\omega}_{\mathcal{M}}\left(h_{1}\left(g_{1}, t_{1}\right) h_{2}\left(g_{2}, t_{2}\right)\right) \\
= & \widetilde{\omega}_{\mathcal{M}}\left(h_{1}\left(g_{1}, t_{1}\right) h_{2}\left(g_{1}, t_{1}\right)^{-1}\left(g_{1}, t_{1}\right)\left(g_{2}, t_{2}\right)\right) \\
= & \widetilde{\omega}_{\mathcal{M}}\left(h_{1}\left(g_{1}, t_{1}\right) h_{2}\left(g_{1}, t_{1}\right)^{-1}\left(g_{1} g_{2}, t_{1} t_{2} c_{\mathcal{M}}\left(g_{1}, g_{2}\right)^{-1}\right)\right) \\
= & t_{1} t_{2} c_{\mathcal{M}}\left(g_{1}, g_{2}\right)^{-1} \mathscr{W}_{\mathcal{M}}\left(h_{1}\left(g_{1}, t_{1}\right) h_{2}\left(g_{1}, t_{1}\right)^{-1}\right) R_{\mathcal{M}}\left(g_{1} g_{2}\right) \\
= & t_{1} t_{2} \mathscr{W}_{\mathcal{M}}\left(h_{1}\right) \mathscr{W}_{\mathcal{M}}\left(\left(g_{1}, t_{1}\right) h_{2}\left(g_{1}, t_{1}\right)^{-1}\right) R_{\mathcal{M}}\left(g_{1}\right) R_{\mathcal{M}}\left(g_{2}\right) \\
= & t_{1} t_{2} \mathscr{W}_{\mathcal{M}}\left(h_{1}\right) \mathscr{W}_{\mathcal{M}}\left(g_{1} h_{2} g_{1}^{-1}\right) R_{\mathcal{M}}\left(g_{1}\right) R_{\mathcal{M}}\left(g_{2}\right) \\
= & t_{1} t_{2} \mathscr{W}_{\mathcal{M}}\left(h_{1}\right) R_{\mathcal{M}}\left(g_{1}\right) \mathscr{W}_{\mathcal{M}}\left(h_{2}\right) R_{\mathcal{M}}\left(g_{2}\right) \\
= & \left\{t_{1} \pi_{\mathcal{M}}\left(h_{1} g_{1}\right)\right\}\left\{t_{2} \pi_{\mathcal{M}}\left(h_{2} g_{2}\right)\right\} \\
= & \widetilde{\omega}_{\mathcal{M}}\left(h_{1}\left(g_{1}, t_{1}\right)\right) \tilde{\omega}_{\mathcal{M}}\left(h_{2}\left(g_{2}, t_{2}\right)\right) .
\end{aligned}
$$

Here we used the fact that $\left(g_{1}, t_{1}\right) h_{2}\left(g_{1}, t_{1}\right)^{-1}=g_{1} h_{2} g_{1}^{-1}$.

We recall that the following matrices

$$
\begin{aligned}
t(b) & =\left(\begin{array}{cc}
I_{n} & b \\
0 & I_{n}
\end{array}\right) \text { with any } b={ }^{t} b \in \mathbb{R}^{(n, n)}, \\
g(\alpha) & =\left(\begin{array}{cc}
{ }^{t} \alpha & 0 \\
0 & \alpha^{-1}
\end{array}\right) \text { with any } \alpha \in G L(n, \mathbb{R}), \\
\sigma_{n} & =\left(\begin{array}{cc}
0 & -I_{n} \\
I_{n} & 0
\end{array}\right)
\end{aligned}
$$


generate the symplectic group $G=S p(n, \mathbb{R})$ (cf. [3, p. 326], [12, p. 210]). Therefore the following elements $h_{t}(\lambda, \mu ; \kappa), t(b ; t), g(\alpha ; t)$ and $\sigma_{n ; t}$ of $G_{\mathcal{M}} \ltimes$ $H_{\mathbb{R}}^{(n, m)}$ defined by

$$
\begin{aligned}
& h_{t}(\lambda, \mu ; \kappa)=\left(\left(I_{2 n}, t\right),(\lambda, \mu ; \kappa)\right) \text { with } t \in T, \lambda, \mu \in \mathbb{R}^{(m, n)} \text { and } \kappa \in \mathbb{R}^{(m, m)}, \\
& t(b ; t)=((t(b), t),(0,0 ; 0)) \text { with any } b={ }^{t} b \in \mathbb{R}^{(n, n)}, t \in T \\
& g(\alpha ; t)=((g(\alpha), t),(0,0 ; 0)) \text { with any } \alpha \in G L(n, \mathbb{R}) \text { and } t \in T, \\
& \sigma_{n ; t}=\left(\left(\sigma_{n}, t\right),(0,0 ; 0)\right) \text { with } t \in T
\end{aligned}
$$

generate the group $G_{\mathcal{M}} \ltimes H_{\mathbb{R}}^{(n, m)}$. We can show that the representation $\widetilde{\omega}_{\mathcal{M}}$ is realized on the representation $H\left(\chi_{\mathcal{M}}\right)=L^{2}\left(\mathbb{R}^{(m, n)}\right)$ as follows: for each $f \in L^{2}\left(\mathbb{R}^{(m, n)}\right)$ and $x \in \mathbb{R}^{(m, n)}$, the actions of $\widetilde{\omega}_{\mathcal{M}}$ on the generators are given by

$$
\begin{aligned}
& {\left[\widetilde{\omega}_{\mathcal{M}}\left(h_{t}(\lambda, \mu ; \kappa)\right) f\right](x)=t e^{\pi i \sigma\left\{\mathcal{M}\left(\kappa+\mu^{t} \lambda+2 x^{t} \mu\right)\right\}} f(x+\lambda),} \\
& {\left[\widetilde{\omega}_{\mathcal{M}}(t(b ; t)) f\right](x)=t e^{\pi i \sigma\left(\mathcal{M} x b^{t} x\right)} f(x),} \\
& {\left[\widetilde{\omega}_{\mathcal{M}}(g(\alpha ; t)) f\right](x)=t|\operatorname{det} \alpha|^{\frac{m}{2}} f\left(x^{t} \alpha\right),} \\
& {\left[\widetilde{\omega}_{\mathcal{M}}\left(\sigma_{n ; t}\right) f\right](x)=t(\operatorname{det} \mathcal{M})^{\frac{n}{2}} \int_{\mathbb{R}^{(m, n)}} f(y) e^{-2 \pi i \sigma\left(\mathcal{M} y^{t} x\right)} d y}
\end{aligned}
$$

Let

$$
G_{2, \mathcal{M}}^{J}=G_{2, \mathcal{M}} \ltimes H_{\mathbb{R}}^{(n, m)}
$$

be the semidirect product of $G_{2, \mathcal{M}}$ and $H_{\mathbb{R}}^{(n, m)}$. Then $G_{2, \mathcal{M}}^{J}$ is a subgroup of $G_{\mathcal{M}}^{J}$ which is a two-fold covering group of the Jacobi group $G^{J}$. The restriction $\omega_{\mathcal{M}}$ of $\widetilde{\omega}_{\mathcal{M}}$ to $G_{2, \mathcal{M}}^{J}$ is called the Schrödinger-Weil representation of $G^{J}$ associated with $\mathcal{M}$.

We denote by $L_{+}^{2}\left(\mathbb{R}^{(m, n)}\right)\left(\right.$ resp. $\left.L_{-}^{2}\left(\mathbb{R}^{(m, n)}\right)\right)$ the subspace of $L^{2}\left(\mathbb{R}^{(m, n)}\right)$ consisting of even (resp. odd) functions in $L^{2}\left(\mathbb{R}^{(m, n)}\right)$. According to Formulas (3.10)-(3.12), $R_{2, \mathcal{M}}$ is decomposed into representations of $R_{2, \mathcal{M}}^{ \pm}$

$$
R_{2, \mathcal{M}}=R_{2, \mathcal{M}}^{+} \oplus R_{2, \mathcal{M}}^{-}
$$

where $R_{2, \mathcal{M}}^{+}$and $R_{2, \mathcal{M}}^{-}$are the even Weil representation and the odd Weil representation of $G$ that are realized on $L_{+}^{2}\left(\mathbb{R}^{(m, n)}\right)$ and $L_{-}^{2}\left(\mathbb{R}^{(m, n)}\right)$ respectively. Obviously the center $\mathscr{Z}_{2, \mathcal{M}}^{J}$ of $G_{2, \mathcal{M}}^{J}$ is given by

$$
\mathscr{Z}_{2, \mathcal{M}}^{J}=\left\{\left(\left(I_{2 n}, 1\right),(0,0 ; \kappa)\right) \in G_{2, \mathcal{M}}^{J}\right\} \cong S(m, \mathbb{R}) .
$$

We note that the restriction of $\omega_{\mathcal{M}}$ to $G_{2, \mathcal{M}}$ coincides with $R_{2, \mathcal{M}}$ and $\omega_{\mathcal{M}}(h)=$ $\mathscr{W}_{\mathcal{M}}(h)$ for all $h \in H_{\mathbb{R}}^{(n, m)}$.

Remark 3.1. In the case $n=m=1, \omega_{\mathcal{M}}$ is dealt in $[1,11]$. We refer to $[4,8]$ for more details about the Weil representation $R_{2, \mathcal{M}}$. 
Remark 3.2. The Schrödinger-Weil representation is applied usefully to the theory of Maass-Jacobi forms [13].

\section{The automorphy factor $J_{1 / 2}$ of Takase}

Let $\rho$ be a rational representation of $G L(n, \mathbb{C})$ on a finite dimensional complex vector space $V_{\rho}$. Let $\mathcal{M} \in \mathbb{R}^{(m, m)}$ be a symmetric half-integral semipositive definite matrix of degree $m$. Let $C^{\infty}\left(\mathbb{H}_{n, m}, V_{\rho}\right)$ be the algebra of all $C^{\infty}$ functions on $\mathbb{H}_{n, m}$ with values in $V_{\rho}$. For $f \in C^{\infty}\left(\mathbb{H}_{n, m}, V_{\rho}\right)$, we define

$$
\begin{aligned}
& \left(\left.f\right|_{\rho, \mathcal{M}}[(g,(\lambda, \mu ; \kappa))]\right)(\Omega, Z) \\
:= & e^{-2 \pi i \sigma\left(\mathcal{M}(Z+\lambda \Omega+\mu)(C \Omega+D)^{-1} C^{t}(Z+\lambda \Omega+\mu)\right)} \times e^{2 \pi i \sigma\left(\mathcal{M}\left(\lambda \Omega^{t} \lambda+2 \lambda^{t} Z+\kappa+\mu^{t} \lambda\right)\right)} \\
& \times \rho(C \Omega+D)^{-1} f\left((A \Omega+B)(C \Omega+D)^{-1},(Z+\lambda \Omega+\mu)(C \Omega+D)^{-1}\right),
\end{aligned}
$$

where $g=\left(\begin{array}{cc}A & B \\ C & D\end{array}\right) \in S p(n, \mathbb{R}),(\lambda, \mu ; \kappa) \in H_{\mathbb{R}}^{(n, m)}$ and $(\Omega, Z) \in \mathbb{H}_{n, m}$.

Definition 4.1. Let $\rho$ and $\mathcal{M}$ be as above. Let

$$
H_{\mathbb{Z}}^{(n, m)}:=\left\{(\lambda, \mu ; \kappa) \in H_{\mathbb{R}}^{(n, m)} \mid \lambda, \mu \in \mathbb{Z}^{(m, n)}, \kappa \in \mathbb{Z}^{(m, m)}\right\}
$$

be the discrete subgroup of $H_{\mathbb{R}}^{(n, m)}$. A Jacobi form of index $\mathcal{M}$ with respect to $\rho$ on a subgroup $\Gamma$ of $\Gamma_{n}$ of finite index is a holomorphic function $f \in$ $C^{\infty}\left(\mathbb{H}_{n, m}, V_{\rho}\right)$ satisfying the following conditions $(\mathrm{A})$ and $(\mathrm{B})$ :

(A) $\left.f\right|_{\rho, \mathcal{M}}[\tilde{\gamma}]=f$ for all $\tilde{\gamma} \in \widetilde{\Gamma}:=\Gamma \ltimes H_{\mathbb{Z}}^{(n, m)}$.

(B) For each $M \in \Gamma_{n},\left.f\right|_{\rho, \mathcal{M}}[M]$ has a Fourier expansion of the following form:

$$
\left(\left.f\right|_{\rho, \mathcal{M}}[M]\right)(\Omega, Z)=\sum_{\substack{T=t_{T} \geq 0 \\ \text { half-integral }}} \sum_{R \in \mathbb{Z}^{(n, m)}} c(T, R) \cdot e^{\frac{2 \pi i}{\lambda^{N}} \sigma(T \Omega)} \cdot e^{2 \pi i \sigma(R Z)}
$$

with a suitable $\lambda_{\Gamma}(\neq 0) \in \mathbb{Z}$ and $c(T, R) \neq 0$ only if $\left(\begin{array}{cc}\frac{1}{\lambda_{\Gamma}} T & \frac{1}{2} R \\ \frac{1}{2}^{t} R & \mathcal{M}\end{array}\right) \geq 0$.

If $n \geq 2$, the condition (B) is superfluous by Köcher principle (cf. [35] Lemma 1.6). We denote by $J_{\rho, \mathcal{M}}(\Gamma)$ the vector space of all Jacobi forms of index $\mathcal{M}$ with respect to $\rho$ on $\Gamma$. Ziegler (cf. [35] Theorem 1.8 or [2] Theorem 1.1) proves that the vector space $J_{\rho, \mathcal{M}}(\Gamma)$ is finite dimensional. In the special case $\rho(A)=(\operatorname{det}(A))^{k}$ with $A \in G L(n, \mathbb{C})$ and a fixed $k \in \mathbb{Z}$, we write $J_{k, \mathcal{M}}(\Gamma)$ instead of $J_{\rho, \mathcal{M}}(\Gamma)$ and call $k$ the weight of the corresponding Jacobi forms. For more results about Jacobi forms with $n>1$ and $m>1$, we refer to $[20,21,23,24,35]$. Jacobi forms play an important role in lifting elliptic cusp forms to Siegel cusp forms of degree $2 n$ (cf. [6]).

Definition 4.2. A Jacobi form $f \in J_{\rho, \mathcal{M}}(\Gamma)$ is said to be a cusp form if $\left(\begin{array}{cc}\frac{1}{\lambda_{\Gamma}} T & \frac{1}{2} R \\ \frac{1}{2}{ }^{t} R & \mathcal{M}\end{array}\right)>0$ for any $T, R$ with $c(T, R) \neq 0$. A Jacobi form $f \in J_{\rho, \mathcal{M}}(\Gamma)$ 
is said to be singular if it admits a Fourier expansion such that a Fourier coefficient $c(T, R)$ vanishes unless $\operatorname{det}\left(\begin{array}{cc}\frac{1}{\lambda_{\Gamma}} T & \frac{1}{2} R \\ \frac{1}{2}{ }^{t} R & \mathcal{M}\end{array}\right)=0$.

Remark 4.1. Singular Jacobi forms were characterized by a certain differential operator and the weight by the author [23].

Without loss of generality we may assume that $\rho$ is irreducible. Then we choose a hermitian inner product $\langle$,$\rangle on V_{\rho}$ that is preserved under the unitary group $U(n) \subset G L(n, \mathbb{C})$. For two Jacobi forms $f_{1}$ and $f_{2}$ in $J_{\rho, \mathcal{M}}(\Gamma)$, putting $\tilde{\Gamma}=\Gamma \ltimes H_{\mathbb{Z}}^{(n, m)}$, we define the Petersson inner product formally by

$$
\left(f_{1}, f_{2}\right):=\int_{\tilde{\Gamma} \backslash \mathbb{H}_{n, m}}\left\langle\rho\left(Y^{\frac{1}{2}}\right) f_{1}(\Omega, Z), \rho\left(Y^{\frac{1}{2}}\right) f_{2}(\Omega, Z)\right\rangle \kappa_{\mathcal{M}}(\Omega, Z) d v .
$$

Here

$$
d v=(\operatorname{det} Y)^{-(n+m+1)}[d X] \wedge[d Y] \wedge[d U] \wedge[d V]
$$

is a $G^{J}$-invariant volume element on $\mathbb{H}_{n, m}$ and

$$
\kappa_{\mathcal{M}}(\Omega, Z):=e^{-4 \pi i \sigma\left({ }^{t}(\operatorname{Im} Z) \mathcal{M} \operatorname{Im} Z(\operatorname{Im} \Omega)^{-1}\right)}=e^{-4 \pi i \sigma\left({ }^{t} V \mathcal{M} V Y^{-1}\right)},
$$

where $\Omega=X+i Y \in \mathbb{H}_{n}, Z=U+i V \in \mathbb{C}^{(m, n)}, X=\left(x_{i j}\right), Y=\left(y_{i j}\right), U=$ $\left(u_{k l}\right), V=\left(v_{k l}\right)$ real and

$$
[d X]=\bigwedge_{i \leq j} d x_{i j}, \quad[d Y]=\bigwedge_{i \leq j} d y_{i j}, \quad[d U]=\bigwedge_{k \leq l} d u_{k l} \quad \text { and } \quad[d V]=\bigwedge_{k \leq l} d v_{k l}
$$

A Jacobi form $f$ in $J_{\rho, \mathcal{M}}(\Gamma)$ is said to be square integrable if $(f, f)<\infty$. We note that cusp Jacobi forms are square integrable and that $\left(f_{1}, f_{2}\right)$ is finite if one of $f_{1}$ and $f_{2}$ is a cusp Jacobi form (cf. [35], p. 203).

For $g=\left(\begin{array}{cc}A & B \\ C & D\end{array}\right) \in G$, we set

$$
J(g, \Omega)=C \Omega+D, \quad \Omega \in \mathbb{H}_{n} .
$$

We define the map $J_{\mathcal{M}}: G^{J} \times \mathbb{H}_{n, m} \longrightarrow \mathbb{C}^{\times}$by

$J_{\mathcal{M}}(\widetilde{g},(\Omega, Z)):=e^{2 \pi i \sigma\left(\mathcal{M}[Z+\lambda \Omega+\mu](C \Omega+D)^{-1} C\right)} \cdot e^{-2 \pi i \sigma\left(\mathcal{M}\left(\lambda \Omega^{t} \lambda+2 \lambda^{t} Z+\kappa+\mu^{t} \lambda\right)\right)}$, where $\widetilde{g}=(g,(\lambda, \mu ; \kappa)) \in G^{J}$ with $g=\left(\begin{array}{cc}A & B \\ C\end{array}\right) \in G$ and $(\lambda, \mu ; \kappa) \in H_{\mathbb{R}}^{(n, m)}$. Here we use the Siegel's notation $S[X]:={ }^{t} X S X$ for two matrices $S$ and $X$.

We define the map $J_{\rho, \mathcal{M}}: G^{J} \times \mathbb{H}_{n, m} \longrightarrow G L\left(V_{\rho}\right)$ by

$$
J_{\rho, \mathcal{M}}(\widetilde{g},(\Omega, Z))=J_{\mathcal{M}}(\widetilde{g},(\Omega, Z)) \rho(J(g, \Omega)),
$$

where $\widetilde{g}=(g, h) \in G^{J}$ with $g \in G$ and $h \in H_{\mathbb{R}}^{(n, m)}$. For a function $f$ on $\mathbb{H}_{n}$ with values in $V_{\rho}$, we can lift $f$ to a function $\Phi_{f}$ on $G^{J}$ :

$$
\begin{aligned}
\Phi_{f}(\sigma): & =\left(\left.f\right|_{\rho, \mathcal{M}}[\sigma]\right)\left(i I_{n}, 0\right) \\
& =J_{\rho, \mathcal{M}}\left(\sigma,\left(i I_{n}, 0\right)\right)^{-1} f\left(\sigma \cdot\left(i I_{n}, 0\right)\right), \quad \sigma \in G^{J} .
\end{aligned}
$$


A characterization of $\Phi_{f}$ for a cusp Jacobi form $f$ in $J_{\rho, \mathcal{M}}(\Gamma)$ was given by Takase [15, pp. 162-164].

We allow a weight $k$ to be half-integral. Let

$$
\mathfrak{S}=\left\{S \in \mathbb{C}^{(n, n)} \mid S={ }^{t} S, \operatorname{Re}(S)>0\right\}
$$

be a connected simply connected complex manifold. Then there is a uniquely determined holomorphic function $\operatorname{det}^{1 / 2}$ on $\mathfrak{S}$ such that

$$
\begin{aligned}
\left(\operatorname{det}^{1 / 2} S\right)^{2} & =\operatorname{det} S \quad \text { for all } S \in \mathfrak{S}, \\
\operatorname{det}^{1 / 2} S & =(\operatorname{det} S)^{1 / 2} \quad \text { for all } S \in \mathfrak{S} \cap \mathbb{R}^{(n, n)} .
\end{aligned}
$$

For each integer $k \in \mathbb{Z}$ and $S \in \mathfrak{S}$, we put

$$
\operatorname{det}^{k / 2} S=\left(\operatorname{det}^{1 / 2} S\right)^{k} \text {. }
$$

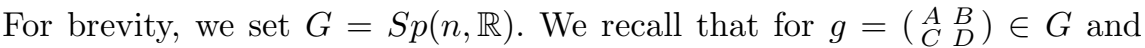
$\Omega \in \mathbb{H}_{n}$

$$
g \cdot \Omega=(A \Omega+B)(C \Omega+D)^{-1} .
$$

For any $g \in G$ and $\Omega, \Omega^{\prime} \in \mathbb{H}_{n}$, we put

$$
\begin{aligned}
\varepsilon\left(g ; \Omega^{\prime}, \Omega\right)= & \operatorname{det}^{-1 / 2}\left(\frac{g \cdot \Omega^{\prime}-\overline{g \cdot \Omega}}{2 i}\right) \operatorname{det}^{1 / 2}\left(\frac{\Omega^{\prime}-\bar{\Omega}}{2 i}\right) \\
& \times\left|\operatorname{det} J\left(g, \Omega^{\prime}\right)\right|^{-1 / 2}|\operatorname{det} J(g, \Omega)|^{-1 / 2} .
\end{aligned}
$$

For each $\Omega \in \mathbb{H}_{n}$, we define the function $\beta_{\Omega}: G \times G \longrightarrow T$ by

$$
\beta_{\Omega}\left(g_{1}, g_{2}\right)=\varepsilon\left(g_{1} ; \Omega, g_{2}(\Omega)\right), \quad g_{1}, g_{2} \in G .
$$

Then $\beta_{\Omega}$ satisfies the cocycle condition and the cohomology class of $\beta_{\Omega}$ is of order two :

$$
\beta_{\Omega}\left(g_{1}, g_{2}\right)^{2}=\alpha_{\Omega}\left(g_{2}\right) \alpha_{\Omega}\left(g_{1} g_{2}\right)^{-1} \alpha_{\Omega}\left(g_{1}\right)
$$

where

$$
\alpha_{\Omega}(g)=\frac{\operatorname{det} J(g, \Omega)}{|\operatorname{det} J(g, \Omega)|}, \quad g \in G, \Omega \in \mathbb{H}_{n}
$$

For any $\Omega \in \mathbb{H}_{n}$, we let

$$
G_{\Omega}=\left\{(g, \epsilon) \in G \times T \mid \epsilon^{2}=\alpha_{\Omega}(g)^{-1}\right\}
$$

be the two-fold covering group with the multiplication law

$$
\left(g_{1}, \epsilon_{1}\right)\left(g_{2}, \epsilon_{2}\right)=\left(g_{1} g_{2}, \epsilon_{1} \epsilon_{2} \beta_{\Omega}\left(g_{1}, g_{2}\right)\right) .
$$

The covering group $G_{\Omega}$ depends on the choice of $\Omega \in \mathbb{H}_{n}$, i.e., the choice of a maximal compact subgroup of $G$. However for any two element $\Omega_{1}, \Omega_{2} \in$ $\mathbb{H}_{n}, G_{\Omega_{1}}$ is isomorphic to $G_{\Omega_{2}}$ (cf. [16]). We put

$$
G_{*}:=G_{i I_{n}} \text {. }
$$


Takase [16, p. 131] defined the automorphy factor $J_{1 / 2}: G_{*} \times \mathbb{H}_{n} \longrightarrow \mathbb{C}^{\times}$by

$$
J_{1 / 2}\left(g_{\epsilon}, \Omega\right):=\epsilon^{-1} \varepsilon\left(g ; \Omega, i I_{n}\right)|\operatorname{det} J(g, \Omega)|^{1 / 2},
$$

where $g_{\epsilon}=(g, \epsilon) \in G_{*}$ with $g \in G$ and $\Omega \in \mathbb{H}_{n}$. It is easily checked that

$$
J_{1 / 2}\left(g_{*} h_{*}, \Omega\right)=J_{1 / 2}\left(g_{*}, h \cdot \Omega\right) J_{1 / 2}\left(h_{*}, \Omega\right)
$$

for all $g_{*}=(g, \epsilon), h_{*}=(h, \eta) \in G_{*}$ and $\Omega \in \mathbb{H}_{n}$. Moreover

$$
J_{1 / 2}\left(g_{*}, \Omega\right)^{2}=\operatorname{det}(C \Omega+D)
$$

for all $g_{*}=(g, \epsilon) \in G_{*}$ with $g=\left(\begin{array}{cc}A & B \\ C & D\end{array}\right) \in G$.

Let $\pi_{*}: G_{*} \longrightarrow G$ be the projection defined by $\pi_{*}(g, \epsilon)=g$. Let $\Gamma$ be a subgroup of the Siegel modular group $\Gamma_{n}$ of finite index. Let $\Gamma_{*}=\pi_{*}^{-1}(\Gamma) \subset G_{*}$. Let $\chi$ be a finite order unitary character of $\Gamma_{*}$. Let $k \in \mathbb{Z}^{+}$be a positive integer. We say that a holomorphic function $\phi: \mathbb{H}_{n} \longrightarrow \mathbb{C}$ is a Siegel modular form of a half-integral weight $k / 2$ with level $\Gamma$ if it satisfies the condition

$$
\phi\left(\gamma_{*} \cdot \Omega\right)=\chi\left(\gamma_{*}\right) J_{1 / 2}\left(\gamma_{*}, \Omega\right)^{k} \phi(\Omega)
$$

for all $\gamma_{*} \in \Gamma_{*}$ and $\Omega \in \mathbb{H}_{n}$ and is holomorphic at all cusps. We denote by $M_{k / 2}(\Gamma, \chi)$ be the vector space of all Siegel modular forms of weight $k / 2$ with level $\Gamma$. Let $S_{k / 2}(\Gamma, \chi)$ be the subspace of $M_{k / 2}(\Gamma, \chi)$ consisting of $\phi \in$ $M_{k / 2}(\Gamma, \chi)$ such that

$$
|\phi(\Omega)| \operatorname{det}(\operatorname{Im} \Omega)^{k / 4} \text { is bounded on } \mathbb{H}_{n} \text {. }
$$

An element of $S_{k / 2}(\Gamma, \chi)$ is called a Siegel cusp form of weight $k / 2$. The Petersson norm on $S_{k / 2}(\Gamma, \chi)$ is defined by

$$
\|\phi\|^{2}=\int_{\Gamma \backslash \mathbb{H}_{n}}|\phi(\Omega)|^{2} \operatorname{det}(\operatorname{Im} \Omega)^{k / 2} d v_{\Omega},
$$

where

$$
d v_{\Omega}=(\operatorname{det} Y)^{-(n+1)}[d X][d Y]
$$

is a $G$-invariant volume element on $\mathbb{H}_{n}$.

Remark 4.2. Using the Schrödinger-Weil representation, Takase [17] established a bijective correspondence between the space of cuspidal Jacobi forms and the space of Siegel cusp forms of half integral weight which is compatible with the action of Hecke operators. For example, if $m$ is a positive integer, the classical result (cf. $[2,5])$

$$
J_{m, 1}^{c u s p}\left(\Gamma_{n}\right) \cong S_{m-1 / 2}\left(\Gamma_{0}(4)\right)
$$

can be obtained by the method of the representation theory. Here $\Gamma_{0}(4)$ is the Hecke subgroup of the Siegel modular group $\Gamma_{n}$ and $J_{m, 1}^{\text {cusp }}\left(\Gamma_{n}\right)$ denotes the vector space of cuspidal Jacobi forms of weight $m$ and index 1. 


\section{Covariant maps for the Schrödinger-Weil representation}

As before we let $\mathcal{M}$ be a positive definite symmetric $m \times m$ real matrix. We keep the notations in the previous sections.

We define the mapping $\mathscr{F}^{(\mathcal{M})}: \mathbb{H}_{n, m} \longrightarrow L^{2}\left(\mathbb{R}^{(m, n)}\right)$ by

$$
\mathscr{F}^{(\mathcal{M})}(\Omega, Z)(x)=e^{\pi i \sigma\left\{\mathcal{M}\left(x \Omega^{t} x+2 x^{t} Z\right)\right\}}, \quad(\Omega, Z) \in \mathbb{H}_{n, m}, x \in \mathbb{R}^{(m, n)} .
$$

For brevity we put $\mathscr{F}_{\Omega, Z}^{(\mathcal{M})}:=\mathscr{F}^{(\mathcal{M})}(\Omega, Z)$ for $(\Omega, Z) \in \mathbb{H}_{n, m}$. Takase [15] proved that $G_{2, \mathcal{M}}$ is isomorphic to $G_{i I_{n}}$ (cf. (3.6) and (4.14)). Therefore we will use $G_{*}:=G_{i I_{n}}$ instead of $G_{2, \mathcal{M}}$.

We set

$$
G_{*}^{J}:=G_{*} \ltimes H_{\mathbb{R}}^{(n, m)} .
$$

We note that $G_{*}^{J}$ acts on $\mathbb{H}_{n, m}$ via the canonical projection of $G_{*}^{J}$ onto $G^{J}$. by

We define the automorphy factor $J_{\mathcal{M}}^{*}: G_{*}^{J} \times \mathbb{H}_{n, m} \longrightarrow \mathbb{C}^{\times}$for $G_{*}^{J}$ on $\mathbb{H}_{n, m}$

$$
\begin{aligned}
J_{\mathcal{M}}^{*}\left(\widetilde{g}_{*},(\Omega, Z)\right)= & e^{\pi i \sigma\left(\mathcal{M}(Z+\lambda \Omega+\mu)(C \Omega+D)^{-1} C^{t}(Z+\lambda \Omega+\mu)\right)} \\
& \times e^{-\pi i \sigma\left(\mathcal{M}\left(\lambda \Omega^{t} \lambda+2 \lambda^{t} Z+\kappa+\mu^{t} \lambda\right)\right)}\left\{J_{1 / 2}((g, \epsilon), \Omega)\right\}^{m},
\end{aligned}
$$

where $\widetilde{g}_{*}=((g, \epsilon),(\lambda, \mu ; \kappa)) \in G_{*}^{J}$ with $g=(\underset{C}{A} \stackrel{B}{D}) \in G,(\lambda, \mu ; \kappa) \in H_{\mathbb{R}}^{(n, m)}$ and $(\Omega, Z) \in \mathbb{H}_{n, m}$.

Theorem 5.1. Let $\mathcal{M}$ be a positive definite symmetric $m \times m$ real matrix. Then the map $\mathscr{F}^{(\mathcal{M})}: \mathbb{H}_{n, m} \longrightarrow L^{2}\left(\mathbb{R}^{(m, n)}\right)$ defined by $(5.1)$ is a covariant map for the Schrödinger-Weil representation $\omega_{\mathcal{M}}$ of $G^{J}$ and the automorphy factor $J_{\mathcal{M}}^{*}$ for $G_{*}^{J}$ on $\mathbb{H}_{n, m}$ defined by Formula (5.3). In other words, $\mathscr{F}(\mathcal{M})$ satisfies the following covariance relation

$$
\omega_{\mathcal{M}}\left(\widetilde{g}_{*}\right) \mathscr{F}_{\Omega, Z}^{(\mathcal{M})}=c\left(\widetilde{g}_{*}\right) J_{\mathcal{M}}^{*}\left(\widetilde{g}_{*},(\Omega, Z)\right)^{-1} \mathscr{F}_{\widetilde{g}_{*} \cdot(\Omega, Z)}^{(\mathcal{M})}
$$

for all $\widetilde{g}_{*} \in G_{*}^{J}$ and $(\Omega, Z) \in \mathbb{H}_{n, m}$. Here $c\left(\widetilde{g}_{*}\right)$ is the constant determined uniquely by $\widetilde{g}_{*}$.

Proof. For an element $\widetilde{g}_{*}=((g, \epsilon),(\lambda, \mu ; \kappa)) \in G_{*}^{J}$ with $g=\left(\begin{array}{cc}A & B \\ C & D\end{array}\right) \in S p(n, \mathbb{R})$, we put $\left(\Omega_{*}, Z_{*}\right)=\widetilde{g}_{*} \cdot(\Omega, Z)$ for $(\Omega, Z) \in \mathbb{H}_{n, m}$. Then we have

$$
\begin{aligned}
& \Omega_{*}=g \cdot \Omega=(A \Omega+B)(C \Omega+D)^{-1}, \\
& Z_{*}=(Z+\lambda \Omega+\mu)(C \Omega+D)^{-1} .
\end{aligned}
$$

In this section we use the notations $t(b)$ and $\sigma_{n}$ in Section 3. Since the following elements $h(\lambda, \mu ; \kappa)_{\epsilon}, t(b ; \epsilon)$ and $\sigma_{n, \epsilon}$ of $G_{*}^{J}$ defined by

$$
\begin{aligned}
h(\lambda, \mu ; \kappa)_{\epsilon} & =\left(\left(I_{2 n}, \epsilon\right),(\lambda, \mu ; \kappa)\right) \quad \text { with } \epsilon= \pm 1, \lambda, \mu \in \mathbb{R}^{(m, n)}, \kappa \in \mathbb{R}^{(m, m)}, \\
t(b ; \epsilon) & =((t(b), \epsilon),(0,0 ; 0)) \quad \text { with } \epsilon= \pm 1, b \in S(n, \mathbb{R}), \\
\sigma_{n, \epsilon} & =\left(\left(\sigma_{n}, \epsilon\right),(0,0 ; 0)\right) \text { with } \epsilon^{2}=(-i)^{n} .
\end{aligned}
$$


generate the group $G_{*}^{J}$, it suffices to prove the covariance relation (5.4) for the above generators.

Case I. $\widetilde{g}_{*}=h(\lambda, \mu ; \kappa)_{\epsilon}$ with $\epsilon= \pm 1$ and $\lambda, \mu \in \mathbb{R}^{(m, n)}, \kappa \in \mathbb{R}^{(m, m)}$.

In this case, we have

$$
\Omega_{*}=\Omega, \quad Z_{*}=Z+\lambda \Omega+\mu .
$$

We put

and

$$
h(\lambda, \mu ; \kappa)_{+}:=\left(\left(I_{2 n}, 1\right),(\lambda, \mu ; \kappa)\right)
$$

$$
h(\lambda, \mu ; \kappa)_{-}:=\left(\left(I_{2 n},-1\right),(\lambda, \mu ; \kappa)\right) .
$$

It is easily seen that according to Formula (4.15), we obtain

$$
J_{1 / 2}\left(\left(I_{2 n}, 1\right), \Omega\right)=1 \quad \text { and } \quad J_{1 / 2}\left(\left(I_{2 n},-1\right), \Omega\right)=-1 .
$$

Therefore we get

$$
J_{\mathcal{M}}^{*}\left(h(\lambda, \mu ; \kappa)_{+},(\Omega, Z)\right)=e^{-\pi i \sigma\left\{\mathcal{M}\left(\lambda \Omega^{t} \lambda+2 \lambda^{t} Z+\kappa+\mu^{t} \lambda\right)\right\}}
$$

and

$$
J_{\mathcal{M}}^{*}\left(h(\lambda, \mu ; \kappa)_{-},(\Omega, Z)\right)=-e^{-\pi i \sigma\left\{\mathcal{M}\left(\lambda \Omega^{t} \lambda+2 \lambda^{t} Z+\kappa+\mu^{t} \lambda\right)\right\}} .
$$

According to Formulas (2.4), (3.8) and (3.9), for $x \in \mathbb{R}^{(m, n)}$,

$$
\begin{aligned}
& {\left[\omega_{\mathcal{M}}\left(h(\lambda, \mu ; \kappa)_{+}\right) \mathscr{F}_{\Omega, Z}^{(\mathcal{M})}\right](x) } \\
= & e^{\pi i \sigma\left\{\mathcal{M}\left(\kappa+\mu^{t} \lambda+2 x^{t} \mu\right)\right\}} \mathscr{F}_{\Omega, Z}^{(\mathcal{M})}(x+\lambda) \\
= & e^{\pi i \sigma\left\{\mathcal{M}\left(\kappa+\mu^{t} \lambda+2 x^{t} \mu\right)\right\}} e^{\pi i \sigma\left\{\mathcal{M}\left((x+\lambda) \Omega^{t}(x+\lambda)+2(x+\lambda)^{t} Z\right)\right\}} .
\end{aligned}
$$

On the other hand, according to Formula (5.5), for $x \in \mathbb{R}^{(m, n)}$,

$$
\begin{aligned}
& J_{\mathcal{M}}^{*}\left(h(\lambda, \mu ; \kappa)_{+},(\Omega, Z)\right)^{-1} \mathscr{F}_{\widetilde{g}_{*} \cdot(\Omega, Z)}^{(\mathcal{M})}(x) \\
= & J_{\mathcal{M}}^{*}\left(h(\lambda, \mu ; \kappa)_{+},(\Omega, Z)\right)^{-1} \mathscr{F}_{\Omega, Z+\lambda \Omega+\mu}^{(\mathcal{M})}(x) \\
= & e^{\pi i \sigma\left\{\mathcal{M}\left(\lambda \Omega^{t} \lambda+2 \lambda^{t} Z+\kappa+\mu^{t} \lambda\right)\right\}} \cdot e^{\pi i \sigma\left\{\mathcal{M}\left(x \Omega^{t} x+2 x^{t}(Z+\lambda \Omega+\mu)\right)\right\}} \\
= & e^{\pi i \sigma\left\{\mathcal{M}\left(\kappa+\mu^{t} \lambda+2 x^{t} \mu\right)\right\}} e^{\pi i \sigma\left\{\mathcal{M}\left((x+\lambda) \Omega^{t}(x+\lambda)+2(x+\lambda)^{t} Z\right)\right\}} .
\end{aligned}
$$

Therefore we prove the covariance relation (5.4) in the case $\widetilde{g}_{*}=h(\lambda, \mu ; \kappa)_{+}$.

Similarly we can prove the covariance relation (5.4) in the $\widetilde{g}_{*}=h(\lambda, \mu ; \kappa)_{-}$. In fact, according to Formulas (2.4), (3.8), (3.9) and (5.6), we obtain the following covariance relation

$$
\begin{aligned}
& {\left[\omega_{\mathcal{M}}\left(h(\lambda, \mu ; \kappa)_{-}\right) \mathscr{F}_{\Omega, Z}(\mathcal{M})\right](x) } \\
= & -e^{\pi i \sigma\left\{\mathcal{M}\left(\kappa+\mu^{t} \lambda+2 x^{t} \mu\right)\right\}} e^{\pi i \sigma\left\{\mathcal{M}\left((x+\lambda) \Omega^{t}(x+\lambda)+2(x+\lambda)^{t} Z\right)\right\}} \\
= & J_{\mathcal{M}}^{*}\left(h(\lambda, \mu ; \kappa)_{-},(\Omega, Z)\right)^{-1} \mathscr{F}_{\widetilde{g}_{*} \cdot(\Omega, Z)}^{(\mathcal{M})}(x) .
\end{aligned}
$$

Case II. $\widetilde{g}_{*}=t(b ; \epsilon)$ with $\epsilon= \pm 1$ and $b \in S(n, \mathbb{R})$. 
In this case, we have

$$
\Omega_{*}=\Omega+b \quad \text { and } \quad Z_{*}=Z .
$$

We put

and

$$
t(b)_{+}=((t(b), 1),(0,0 ; 0))
$$

$$
t(b)_{-}=((t(b),-1),(0,0 ; 0)) .
$$

It is easily seen that

$$
J_{1 / 2}((t(b), 1), \Omega)=1 \quad \text { and } \quad J_{1 / 2}((t(b),-1), \Omega)=-1 .
$$

Therefore we get

$$
J_{\mathcal{M}}^{*}\left(t(b)_{+},(\Omega, Z)\right)=1 \quad \text { and } \quad J_{\mathcal{M}}^{*}\left(t(b)_{-},(\Omega, Z)\right)=-1 .
$$

According to Formulas (3.8) and (3.10), we obtain

$$
\left[\omega_{\mathcal{M}}\left(t(b)_{+}\right) \mathscr{F}_{\Omega, Z}^{(\mathcal{M})}\right](x)=e^{\pi i \sigma\left(\mathcal{M} x b^{t} x\right)} \mathscr{F}_{\Omega, Z}^{(\mathcal{M})}(x), \quad x \in \mathbb{R}^{(m, n)} .
$$

On the other hand, according to Formula (5.7), for $x \in \mathbb{R}^{(m, n)}$, we obtain

$$
\begin{aligned}
J_{\mathcal{M}}^{*}\left(t(b)_{+},(\Omega, Z)\right)^{-1} \mathscr{F}_{t(b)_{+} \cdot(\Omega, Z)}^{(\mathcal{M})}(x) & =\mathscr{F}_{\Omega+b, Z}^{(\mathcal{M})}(x) \\
& =e^{\pi i \sigma\left(\mathcal{M}\left(x(\Omega+b)^{t} x+2 x^{t} Z\right)\right)} \\
& =e^{\pi i \sigma\left(\mathcal{M} x b^{t} x\right)} \mathscr{F}_{\Omega, Z}^{(\mathcal{M})}(x) .
\end{aligned}
$$

Therefore we prove the covariance relation (5.4) in the case $\widetilde{g}_{*}=t(b)_{+}$with $b \in S(n, \mathbb{R})$.

Similarly we can prove the covariance relation $(5.4)$ in the $\widetilde{g}_{*}=t(b)_{-}$. In fact, according to Formula (5.7), we obtain the following covariance relation

$$
\begin{aligned}
{\left[\omega_{\mathcal{M}}\left(t(b)_{-}\right) \mathscr{F}_{\Omega, Z}^{(\mathcal{M})}\right](x) } & =-e^{\pi i \sigma\left(\mathcal{M} x b^{t} x\right)} \mathscr{F}_{\Omega, Z}^{(\mathcal{M})}(x) \\
& =J_{\mathcal{M}}^{*}\left(t(b)_{-},(\Omega, Z)\right)^{-1} \mathscr{F}_{t(b)_{-} \cdot(\Omega, Z)}^{(\mathcal{M})}(x) .
\end{aligned}
$$

Case III. $\widetilde{g}_{*}=\left(\left(\sigma_{n}, \epsilon\right),(0,0 ; 0)\right)$ with $\sigma_{n}=\left(\begin{array}{cc}0 & -I_{n} \\ I_{n} & 0\end{array}\right)$ and $\epsilon^{2}=(-i)^{n}$.

In this case, we have

$$
\Omega_{*}=-\Omega^{-1} \quad \text { and } \quad Z_{*}=Z \Omega^{-1} .
$$

In order to prove the covariance relation (5.4), we need the following useful lemma.

Lemma 5.1. For a fixed element $\Omega \in \mathbb{H}_{n}$ and a fixed element $Z \in \mathbb{C}^{(m, n)}$, we obtain the following property

$$
\int_{\mathbb{R}^{(m, n)}} e^{\pi i \sigma\left(x \Omega^{t} x+2 x^{t} Z\right)} d x_{11} \cdots d x_{m n}=\left(\operatorname{det} \frac{\Omega}{i}\right)^{-\frac{m}{2}} e^{-\pi i \sigma\left(Z \Omega^{-1 t} Z\right)},
$$

where $x=\left(x_{i j}\right) \in \mathbb{R}^{(m, n)}$. 
Proof of Lemma 5.1. By a simple computation, we see that

$$
e^{\pi i \sigma\left(x \Omega^{t} x+2 x^{t} Z\right)}=e^{-\pi i \sigma\left(Z \Omega^{-1 t} Z\right)} \cdot e^{\pi i \sigma\left\{\left(x+Z \Omega^{-1}\right) \Omega^{t}\left(x+Z \Omega^{-1}\right)\right\}} .
$$

Since the real Jacobi group $S p(n, \mathbb{R}) \ltimes H_{\mathbb{R}}^{(n, m)}$ acts on $\mathbb{H}_{n, m}$ transitively and holomorphically, we may put

$$
\Omega=i A^{t} A, \quad Z=i V, \quad A \in \mathbb{R}^{(n, n)}, \quad V=\left(v_{i j}\right) \in \mathbb{R}^{(m, n)} .
$$

Then we obtain

$$
\begin{aligned}
& \int_{\mathbb{R}^{(m, n)}} e^{\pi i \sigma\left(x \Omega^{t} x+2 x^{t} Z\right)} d x_{11} \cdots d x_{m n} \\
= & e^{-\pi i \sigma\left(Z \Omega^{-1 t} Z\right)} \int_{\mathbb{R}^{(m, n)}} e^{\pi i \sigma\left[\left\{x+i V\left(i A^{t} A\right)^{-1}\right\}\left(i A^{t} A\right)^{t}\left\{x+i V\left(i A^{t} A\right)^{-1}\right\}\right]} d x_{11} \cdots d x_{m n} \\
= & e^{-\pi i \sigma\left(Z \Omega^{-1 t} Z\right)} \int_{\mathbb{R}^{(m, n)}} e^{\pi i \sigma\left[\left\{x+V\left(A^{t} A\right)^{-1}\right\} A^{t} A^{t}\left\{x+V\left(A^{t} A\right)^{-1}\right\}\right]} d x_{11} \cdots d x_{m n} \\
= & e^{-\pi i \sigma\left(Z \Omega^{-1}{ }^{t} Z\right)} \int_{\mathbb{R}^{(m, n)}} e^{-\pi \sigma\left\{(u A)^{t}(u A)\right\}} d u_{11} \cdots d u_{m n} \\
& \left(\operatorname{put} u=x+V\left(A^{t} A\right)^{-1}=\left(u_{i j}\right)\right) \\
= & e^{-\pi i \sigma\left(Z \Omega^{-1 t} Z\right)} \int_{\mathbb{R}^{(m, n)}} e^{-\pi \sigma\left(w^{t} w\right)}(\operatorname{det} A)^{-m} d w_{11} \cdots d w_{m n} \\
& \left(\operatorname{put} w=u A=\left(w_{i j}\right)\right) \\
= & e^{-\pi i \sigma\left(Z \Omega^{-1 t} Z\right)}(\operatorname{det} A)^{-m} \cdot\left(\prod_{i=1}^{m} \prod_{j=1}^{g} \int_{\mathbb{R}} e^{-\pi w_{i j}^{2}} d w_{i j}\right) \\
= & e^{-\pi i \sigma\left(Z \Omega^{-1 t} Z\right)}(\operatorname{det} A)^{-m}\left(\operatorname{because} \int_{\mathbb{R}} e^{-\pi w_{i j}^{2}} d w_{i j}=1 \quad \text { for all } i, j\right) \\
= & e^{-\pi i \sigma\left(Z \Omega^{-1 t} Z\right)}\left(\operatorname{det}\left(A^{t} A\right)\right)^{-\frac{m}{2}} \\
= & e^{-\pi i \sigma\left(Z \Omega^{-1 t} Z\right)}\left(\operatorname{det}\left(\frac{\Omega^{\prime}}{i}\right)\right)^{-\frac{m}{2}} \cdot
\end{aligned}
$$

This completes the proof of Lemma 5.1.

According to Formulas (3.8) and (3.12), for $x \in \mathbb{R}^{(m, n)}$, we obtain

$$
\begin{aligned}
& {\left[\omega_{\mathcal{M}}\left(\widetilde{g}_{*}\right) \mathscr{F}_{\Omega, Z}^{(\mathcal{M})}\right](x) } \\
= & \epsilon(\operatorname{det} \mathcal{M})^{\frac{n}{2}} \int_{\mathbb{R}^{(m, n)}} \mathscr{F}_{\Omega, Z}^{(\mathcal{M})}(y) e^{-2 \pi i \sigma\left(\mathcal{M} y^{t} x\right)} d y \\
= & \epsilon(\operatorname{det} \mathcal{M})^{\frac{n}{2}} \int_{\mathbb{R}^{(m, n)}} e^{\pi i \sigma\left\{\mathcal{M}\left(y \Omega^{t} y+2 y^{t} Z\right)\right\}} e^{-2 \pi i \sigma\left(\mathcal{M} y^{t} x\right)} d y \\
= & \epsilon(\operatorname{det} \mathcal{M})^{\frac{n}{2}} \int_{\mathbb{R}^{(m, n)}} e^{\pi i \sigma\left\{\mathcal{M}\left(y \Omega^{t} y+2 y^{t}(Z-x)\right)\right\}} d y .
\end{aligned}
$$


If we substitute $u=\mathcal{M}^{\frac{1}{2}} y$, then $d u=(\operatorname{det} \mathcal{M})^{\frac{n}{2}} d y$. Therefore according to Lemma 5.1, we obtain

$$
\begin{aligned}
& {\left[\omega_{\mathcal{M}}\left(\widetilde{g}_{*}\right) \mathscr{F}_{\Omega, Z}^{(\mathcal{M})}\right](x) } \\
= & \epsilon(\operatorname{det} \mathcal{M})^{\frac{n}{2}} \int_{\mathbb{R}^{(m, n)}} e^{\pi i \sigma\left(u \Omega^{t} u+2 \mathcal{M}^{1 / 2} u^{t}(Z-x)\right)}(\operatorname{det} \mathcal{M})^{-\frac{n}{2}} d u \\
= & \epsilon \int_{\mathbb{R}^{(m, n)}} e^{\pi i \sigma\left(u \Omega^{t} u+2 u^{t}\left(\mathcal{M}^{1 / 2}(Z-x)\right)\right)} d u \\
= & \epsilon\left(\operatorname{det} \frac{\Omega}{i}\right)^{-\frac{m}{2}} e^{-\pi i \sigma\left\{\mathcal{M}^{1 / 2}(Z-x) \Omega^{-1 t}(Z-x) \mathcal{M}^{1 / 2}\right\}} \quad \text { (by Lemma 5.1) } \\
= & \epsilon i^{\frac{m n}{2}}(\operatorname{det} \Omega)^{-\frac{m}{2}} e^{-\pi i \sigma\left(\mathcal{M}(Z-x) \Omega^{-1 t}(Z-x)\right)} \\
= & \epsilon i^{\frac{m n}{2}}(\operatorname{det} \Omega)^{-\frac{m}{2}} e^{-\pi i \sigma\left(\mathcal{M}\left(Z \Omega^{-1 t} Z+x \Omega^{-1 t} x-2 Z \Omega^{-1 t} x\right)\right) .}
\end{aligned}
$$

On the other hand,

$$
\begin{aligned}
& J_{1 / 2}\left(\left(\sigma_{n}, \epsilon\right), \Omega\right) \\
= & \epsilon^{-1} \operatorname{det}^{-1 / 2}\left(\frac{\sigma_{n} \cdot \Omega-\overline{\sigma_{n} \cdot\left(i I_{n}\right)}}{2 i}\right) \operatorname{det}^{1 / 2}\left(\frac{\Omega-\overline{\left(i I_{n}\right)}}{2 i}\right)\left|J\left(\sigma_{n}, i I_{n}\right)\right|^{-1 / 2} \\
= & \epsilon^{-1} \operatorname{det}^{-1 / 2}\left(\frac{i \Omega^{-1}\left(\Omega-\overline{i I_{n}}\right)}{2 i}\right) \operatorname{det}^{1 / 2}\left(\frac{\Omega-\overline{\left(i I_{n}\right)}}{2 i}\right) \\
= & \epsilon^{-1} \operatorname{det}^{-1 / 2}\left(i \Omega^{-1}\right) \\
= & \epsilon^{-1}\left(\frac{1}{i}\right)^{n / 2}(\operatorname{det} \Omega)^{1 / 2} .
\end{aligned}
$$

Therefore, according to Formula (5.3), for $x \in \mathbb{R}^{(m, n)}$, we obtain

$$
\begin{aligned}
& J_{\mathcal{M}}^{*}\left(\widetilde{g}_{*},(\Omega, Z)\right)^{-1} \mathscr{F}_{\widetilde{g}_{*} \cdot(\Omega, Z)}^{(\mathcal{M})}(x) \\
= & e^{-\pi i \sigma\left(\mathcal{M} Z \Omega^{-1 t} Z\right)} J_{1 / 2}\left(\left(\sigma_{n}, \epsilon\right), \Omega\right)^{-m} \mathscr{F}_{-\Omega^{-1}, Z \Omega^{-1}}^{(\mathcal{M})}(x) \\
= & \epsilon^{m} i^{\frac{m n}{2}}(\operatorname{det} \Omega)^{-\frac{m}{2}} e^{-\pi i \sigma\left(\mathcal{M} Z \Omega^{-1 t} Z\right)} e^{\pi i \sigma\left\{\mathcal{M}\left(x\left(-\Omega^{-1}\right)^{t} x+2 x^{t}\left(Z \Omega^{-1}\right)\right)\right\}} \\
= & \epsilon^{m} i^{\frac{m n}{2}}(\operatorname{det} \Omega)^{-\frac{m}{2}} e^{-\pi i \sigma\left(\mathcal{M}\left(Z \Omega^{-1 t} Z+x \Omega^{-1 t} x-2 Z \Omega^{-1 t} x\right)\right)} .
\end{aligned}
$$

Hence we prove the covariance relation (5.4) in the case $\widetilde{g}_{*}=\left(\left(\sigma_{n}, \epsilon\right),(0,0 ; 0)\right)$ with $\epsilon^{2}=(-i)^{n}$. Since $J_{\mathcal{M}}^{*}$ is an automorphy factor for $G_{*}^{J}$ on $\mathbb{H}_{n, m}$, we see that if the covariance relation (5.4) holds for two elements $\widetilde{g}_{*}, \widetilde{h}_{*}$ in $G_{*}^{J}$, then it holds for $\widetilde{g}_{*} \widetilde{h}_{*}$. Finally we complete the proof.

Let $\left(\pi, V_{\pi}\right)$ be a unitary representation of $G_{*}^{J}$ on the representation space $V_{\pi}$. Let $\Gamma$ be an arithmetic subgroup of the Siegel modular group $\Gamma_{n}$. We set $\Gamma_{*}=\pi_{*}^{-1}(\Gamma)$ and

$$
\Gamma_{*}^{J}=\Gamma_{*} \ltimes H_{\mathbb{Z}}^{(n, m)} .
$$


We assume that $\left(\pi, V_{\pi}\right)$ satisfies the following conditions (A) and (B):

(A) There exists a vector valued map

$$
\mathscr{F}: \mathbb{H}_{n, m} \longrightarrow V_{\pi}, \quad(\Omega, Z) \mapsto \mathscr{F}_{\Omega, Z}:=\mathscr{F}(\Omega, Z)
$$

satisfying the following covariance relation

$\pi\left(\widetilde{g}_{*}\right) \mathscr{F}_{\Omega, Z}=\psi\left(\widetilde{g}_{*}\right) J_{*}\left(\widetilde{g}_{*},(\Omega, Z)\right)^{-1} \mathscr{F}_{\widetilde{g}_{*} \cdot(\Omega, Z)}$ for all $\widetilde{g}_{*} \in G_{*}^{J},(\Omega, Z) \in \mathbb{H}_{n, m}$, where $\psi$ is a character of $G_{*}^{J}$ and $J_{*}: G_{*}^{J} \times \mathbb{H}_{n, m} \longrightarrow \mathbb{C}^{\times}$is a certain automorphy factor for $G_{*}^{J}$ on $\mathbb{H}_{n, m}$.

(B) There exists a linear functional $\theta: V_{\pi} \longrightarrow \mathbb{C}$ which is semi-invariant under the action of $\Gamma_{*}^{J}$, in other words, for all $\widetilde{\gamma}_{*} \in \Gamma_{*}^{J}$ and $(\Omega, Z) \in \mathbb{H}_{n, m}$,

$$
\left\langle\pi^{*}\left(\widetilde{\gamma}_{*}\right) \theta, \mathscr{F}_{\Omega, Z}\right\rangle=\left\langle\theta, \pi\left(\widetilde{\gamma}_{*}\right)^{-1} \mathscr{F}_{\Omega, Z}\right\rangle=\chi\left(\widetilde{\gamma}_{*}\right)\left\langle\theta, \mathscr{F}_{\Omega, Z}\right\rangle,
$$

where $\pi^{*}$ is the contragredient of $\pi$ and $\chi: \Gamma_{*}^{J} \longrightarrow T$ is a unitary character of $\Gamma_{*}^{J}$.

Under the assumptions (A) and (B) on a unitary representation $\left(\pi, V_{\pi}\right)$, we define the function $\Theta$ on $\mathbb{H}_{n, m}$ by

$$
\Theta(\Omega, Z):=\left\langle\theta, \mathscr{F}_{\Omega, Z}\right\rangle=\theta\left(\mathscr{F}_{\Omega, Z}\right), \quad(\Omega, Z) \in \mathbb{H}_{n, m} .
$$

We now shall see that $\Theta$ is an automorphic form on $\mathbb{H}_{n, m}$ with respect to $\Gamma_{*}^{J}$ for the automorphy factor $J_{*}$.

Lemma 5.2. Let $\left(\pi, V_{\pi}\right)$ be a unitary representation of $G_{*}^{J}$ satisfying the above assumptions $(\mathrm{A})$ and $(\mathrm{B})$. Then the function $\Theta$ on $\mathbb{H}_{n, m}$ defined by (5.11) satisfies the following modular transformation behavior

$$
\Theta\left(\widetilde{\gamma}_{*} \cdot(\Omega, Z)\right)=\psi\left(\widetilde{\gamma}_{*}\right)^{-1} \chi\left(\widetilde{\gamma}_{*}\right)^{-1} J_{*}\left(\widetilde{\gamma}_{*},(\Omega, Z)\right) \Theta(\Omega, Z)
$$

for all $\widetilde{\gamma}_{*} \in \Gamma_{*}^{J}$ and $(\Omega, Z) \in \mathbb{H}_{n, m}$.

Proof. For any $\widetilde{\gamma}_{*} \in \Gamma_{*}^{J}$ and $(\Omega, Z) \in \mathbb{H}_{n, m}$, according to the assumptions (5.9) and (5.10), we obtain

$$
\begin{aligned}
\Theta\left(\widetilde{\gamma}_{*} \cdot(\Omega, Z)\right) & =\left\langle\theta, \mathscr{F} \widetilde{\gamma}_{*} \cdot(\Omega, Z)\right\rangle \\
& =\left\langle\theta, \psi\left(\widetilde{\gamma}_{*}\right)^{-1} J_{*}\left(\widetilde{\gamma}_{*},(\Omega, Z)\right) \pi\left(\widetilde{\gamma}_{*}\right) \mathscr{F}_{\Omega, Z}\right\rangle \\
& =\psi\left(\widetilde{\gamma}_{*}\right)^{-1} J_{*}\left(\widetilde{\gamma}_{*},(\Omega, Z)\right)\left\langle\theta, \pi\left(\widetilde{\gamma}_{*}\right) \mathscr{F}_{\Omega, Z}\right\rangle \\
& =\psi\left(\widetilde{\gamma}_{*}\right)^{-1} \chi\left(\widetilde{\gamma}_{*}\right)^{-1} J_{*}\left(\widetilde{\gamma}_{*},(\Omega, Z)\right)\left\langle\theta, \mathscr{F}_{\Omega, Z}\right\rangle \\
& =\psi\left(\widetilde{\gamma}_{*}\right)^{-1} \chi\left(\widetilde{\gamma}_{*}\right)^{-1} J_{*}\left(\widetilde{\gamma}_{*},(\Omega, Z)\right) \Theta(\Omega, Z) .
\end{aligned}
$$

Now for a positive definite integral symmetric matrix $\mathcal{M}$ of degree $m$, we define the holomorphic function $\Theta_{\mathcal{M}}: \mathbb{H}_{n, m} \longrightarrow \mathbb{C}$ by

$$
\Theta_{\mathcal{M}}(\Omega, Z):=\sum_{\xi \in \mathbb{Z}^{(m, n)}} e^{\pi i \sigma\left(\mathcal{M}\left(\xi \Omega^{t} \xi+2 \xi^{t} Z\right)\right)}, \quad(\Omega, Z) \in \mathbb{H}_{n, m} .
$$


Theorem 5.2. Let $m$ be an odd positive integer. Let $\mathcal{M}$ be a symmetric positive definite integral matrix of degree $m$ such that $\operatorname{det} \mathcal{M}=1$. Let $\Gamma$ be an arithmetic subgroup of $\Gamma_{n}$ generated by all the following elements

$$
t(b)=\left(\begin{array}{cc}
I_{n} & b \\
0 & I_{n}
\end{array}\right), \quad g(\alpha)=\left(\begin{array}{cc}
{ }^{t} \alpha & 0 \\
0 & \alpha^{-1}
\end{array}\right), \quad \sigma_{n}=\left(\begin{array}{cc}
0 & -I_{n} \\
I_{n} & 0
\end{array}\right),
$$

where $b={ }^{t} b \in S(n, \mathbb{Z})$ with even diagonal and $\alpha \in G L(n, \mathbb{Z})$. Then for any $\widetilde{\gamma}_{*} \in \Gamma_{*}^{J}$, the function $\Theta_{\mathcal{M}}$ defined by (5.12) satisfies the functional equation

$$
\Theta_{\mathcal{M}}\left(\widetilde{\gamma}_{*} \cdot(\Omega, Z)\right)=\rho_{\mathcal{M}}\left(\widetilde{\gamma}_{*}\right) J_{\mathcal{M}}^{*}\left(\widetilde{\gamma}_{*},(\Omega, Z)\right) \Theta_{\mathcal{M}}(\Omega, Z), \quad(\Omega, Z) \in \mathbb{H}_{n, m},
$$

where $J_{\mathcal{M}}^{*}: G_{*}^{J} \times \mathbb{H}_{n, m} \longrightarrow \mathbb{C}^{\times}$is the automorphy factor for $G_{*}^{J}$ on $\mathbb{H}_{n, m}$ defined by the formula (5.3) and $\rho_{\mathcal{M}}$ is the character of $\Gamma_{*}^{J}$ determined uniquely by the following formulas

$$
\begin{aligned}
\rho_{\mathcal{M}}\left(\left(\left(I_{2 n}, 1\right),(\lambda, \mu ; \kappa)\right)\right) & =e^{-\pi i \sigma\left(\mathcal{M}\left(\kappa+\mu^{t} \lambda\right)\right)} \quad \text { with } \lambda, \mu, \kappa \text { integral, } \\
\rho_{\mathcal{M}}\left(\left(\left(I_{2 n},-1\right),(\lambda, \mu ; \kappa)\right)\right) & =-e^{-\pi i \sigma\left(\mathcal{M}\left(\kappa+\mu^{t} \lambda\right)\right)} \quad \text { with } \lambda, \mu, \kappa \text { integral, } \\
\rho_{\mathcal{M}}(((t(b), 1),(0,0 ; 0))) & =1 \text { with } b \in S(n, \mathbb{Z}) \text { even diagonal, } \\
\rho_{\mathcal{M}}(((t(b),-1),(0,0 ; 0))) & =-1 \text { with } b \in S(n, \mathbb{Z}) \text { even diagonal, } \\
\rho_{\mathcal{M}}(((g(\alpha), \epsilon),(0,0 ; 0))) & =\epsilon^{m}(\operatorname{det} \alpha)^{\frac{m}{2}} \text { with } \alpha \in G L(n, \mathbb{Z}), \epsilon= \pm 1, \pm i, \\
\rho_{\mathcal{M}}\left(\left(\left(\sigma_{n}, \epsilon\right),(0,0 ; 0)\right)\right) & =\epsilon^{m}(-i)^{\frac{m n}{2}} \text { with } \epsilon^{2}=(-i)^{n} .
\end{aligned}
$$

Proof. The assertion follows from Theorem 5.1 and Lemma 5.2. We leave the detail to the reader.

\section{References}

[1] R. Berndt and R. Schmidt, Elements of the Representation Theory of the Jacobi Group, Birkhäuser, 1998.

[2] M. Eichler and D. Zagier, The Theory of Jacobi Forms, Progress in Math., 55, Birkhäuser, Boston, Basel and Stuttgart, 1985.

[3] E. Freitag, Siegelsche Modulfunktionen, Grundlehren de mathematischen Wissenschaften 55, Springer-Verlag, Berlin-Heidelberg-New York, 1983.

[4] S. Gelbart, Weil's Representation and the Spectrum of the Metaplectic Group, Lecture Notes in Math. 530, Springer-Verlag, Berlin and New York, 1976.

[5] T. Ibukiyama, On Jacobi forms and Siegel modular forms of half integral weights, Comment. Math. Univ. St. Paul. 41 (1992), no. 2, 109-124.

[6] T. Ikeda, On the lifting of elliptic cusp forms to Siegel cusp forms of degree 2n, Ann. of Math. 154 (2001), no. 3, 641-681.

[7] M. Itoh, H. Ochiai, and J.-H. Yang, Invariant differential operators on Siegel-Jacobi space, preprint, 2013.

[8] M. Kashiwara and M. Vergne, On the Segal-Shale-Weil representations and harmonic polynomials, Invent. Math. 44 (1978), no. 1, 1-47.

[9] G. Lion and M. Vergne, The Weil representation, Maslov index and Theta series, Progress in Math., 6, Birkhäuser, Boston, Basel and Stuttgart, 1980.

[10] G. W. Mackey, Induced representations of locally compact groups. I, Ann. of Math. 55 (1952), 101-139.

[11] J. Marklof, Pair correlation densities of inhomogeneous quadratic forms, Ann. of Math. 158 (2003), no. 2, 419-471. 
[12] D. Mumford, Tata Lectures on Theta. I, Progress in Math. 28, Boston-Basel-Stuttgart, 1983.

[13] A. Pitale, Jacobi Maass forms, Abh. Math. Sem. Univ. Hamburg 79 (2009), no. 1, 87-111.

[14] C. L. Siegel, Indefinite quadratische Formen und Funnktionentheorie I and II, Math. Ann. 124 (1951), 17-54 and Math. Ann. 124 (1952), 364-387; Gesammelte Abhandlungen, Band III, Springer-Verlag (1966), 105-142 and 154-177.

[15] K. Takase, A note on automorphic forms, J. Reine Angew. Math. 409 (1990), 138-171.

[16] - On two-fold covering group of $S p(n, \mathbb{R})$ and automorphic factor of weight $1 / 2$, Comment. Math. Univ. St. Paul. 45 (1996), no. 2, 117-145.

[17] - On Siegel modular forms of half-integral weights and Jacobi forms, Trans. Amer. Math. Soc. 351 (1999), no. 2, 735-780.

[18] A. Weil, Sur certains groupes d'operateurs unitares, Acta Math. 111 (1964), 143-211; Collected Papers (1964-1978), Vol. III, Springer-Verlag (1979), 1-69.

[19] J.-H. Yang, Harmonic analysis on the quotient spaces of Heisenberg groups, Nagoya Math. J. 123 (1991), 103-117.

[20] - Remarks on Jacobi forms of higher degree, Proc. of the 1993 Workshop on automorphic forms and related topics (Seoul, 1993), 33-58, Pyungsan Inst. Math. Sci., Seoul, 1993.

[21] The Siegel-Jacobi Operator, Abh. Math. Sem. Univ. Hamburg 63 (1993), 135146.

[22] _ Harmonic analysis on the quotient spaces of Heisenberg groups. II, J. Number Theory 49 (1994), no. 1, 63-72.

[23] _ Singular Jacobi forms, Trans. Amer. Math. Soc. 347 (1995), no. 6, 2041-2049.

[24] Construction of vector valued modular forms from Jacobi forms, Canad. J. Math. 47 (1995), no. 6, 1329-1339.

[25] A A decomposition theorem on differential polynomials of theta functions of high level, Japan. J. Math. (N.S.) 22 (1996), no. 1, 37-49.

[26] - Fock representations of the Heisenberg group $H_{\mathbb{R}}^{(g, h)}$, J. Korean Math. Soc. 34 (1997), no. 2, 345-370.

[27] L Lattice representations of Heisenberg groups, Math. Ann. 317 (2000), no. 2, 309-323.

[28] _ A note on a fundamental domain for Siegel-Jacobi space, Houston J. Math. 32 (2006), no. 3, 701-712.

[29] _ Invariant metrics and Laplacians on Siegel-Jacobi space, J. Number Theory 127 (2007), no. 1, 83-102

[30] _ A partial Cayley transform of Siegel-Jacobi disk, J. Korean Math. Soc. 45 (2008), no. 3, 781-794.

[31] , Invariant metrics and Laplacians on Siegel-Jacobi disk, Chin. Ann. Math. Ser. B 31 (2010), no. 1, 85-100.

[32] _ Heisenberg Group, Theta Functions and the Weil Representation, Kyung Moon Sa, Seoul, 2012.

[33] _ A note on Maass-Jacobi forms II, Kyungpook Math. J. 53 (2013), no. 1, 49-86.

[34] J.-H. Yang, Y.-H. Yong, S.-N. Huh, J.-H. Shin, and G.-H. Min, Sectional curvatures of the Siegel-Jacobi space, Bull. Korean Math. Soc. 50 (2013), no. 3, 787-799.

[35] C. Ziegler, Jacobi forms of higher degree, Abh. Math. Sem. Univ. Hamburg 59 (1989), 191-224.

Department of Mathematics

INHA UNIVERSITY

INCHEON 402-751, KOREA

E-mail address: jhyang@inha.ac.kr 